\title{
Delay Bound: Fractal Traffic Passes through Network Servers
}

\author{
Ming Li, ${ }^{1}$ Wei Zhao, ${ }^{2}$ and Carlo Cattani ${ }^{3}$ \\ ${ }^{1}$ School of Information Science \& Technology, East China Normal University, No. 500 Dongchuan Road, Shanghai 200241, China \\ ${ }^{2}$ Department of Computer and Information Science, University of Macau, Avenida Padre Tomas Pereira, Taipa 1356, Macau \\ ${ }^{3}$ Department of Mathematics, University of Salerno, Via Ponte Don Melillo, 84084 Fisciano, Italy
}

Correspondence should be addressed to Ming Li; ming_lihk@yahoo.com

Received 25 August 2013; Accepted 5 September 2013

Academic Editor: Ezzat G. Bakhoum

Copyright (C) 2013 Ming Li et al. This is an open access article distributed under the Creative Commons Attribution License, which permits unrestricted use, distribution, and reproduction in any medium, provided the original work is properly cited.

Delay analysis plays a role in real-time systems in computer communication networks. This paper gives our results in the aspect of delay analysis of fractal traffic passing through servers. There are three contributions presented in this paper. First, we will explain the reasons why conventional theory of queuing systems ceases in the general sense when arrival traffic is fractal. Then, we will propose a concise method of delay computation for hard real-time systems as shown in this paper. Finally, the delay computation of fractal traffic passing through severs is presented.

\section{Introduction}

There are two categories of communications to perform the delivery of a message $\mathrm{M}$ from the source $\mathrm{A}$ to the destination B. One is in the sense of best effort. By best effort, one means that the computer communication system, which is denoted by $S$, does not guarantee the connection of sending $\mathrm{M}$ from $\mathrm{A}$ to $\mathrm{B}$, and accordingly, the quantity of the time delay $D$ that $M$ suffers from $S$ may not be guaranteed, generally. User Datagram Protocol (UDP) is used for communications by best effort (Tanenbaum [1], Postel [2]). The other is in the sense of Transmission Control Protocol (TCP), which is connection oriented, implying that the connection for sending $\mathrm{M}$ from $\mathrm{A}$ to $\mathrm{B}$ is guaranteed ([1], Postel [3]). Guaranteed connection is the premise for guaranteeing the quantity of the time delay $D$ that $M$ suffers from $S$ from $A$ to $B$. This is particularly the case when mission critical applications are required (Zhao and Ramamritham [4], Zhao et al. [5], Zhao and Stankovic [6], Mahapatra and Zhao [7], Rader [8], and Mahmoodi et al. [9]).

In the case of guaranteed connections, there are two types of communication systems. One is in the type of realtime systems. The other is in the type of nonreal-time ones. By real-time system, one implies that the predetermined time delay should be guaranteed (Natarajan and Zhao [10], Chakraborty and Eberspcher [11]). If the delay, which M suffers from $\mathrm{S}$, exceeds the predetermined deadline of delay, one will consider that the message $M$ is meaningless, and communication of $\mathrm{M}$ from $\mathrm{A}$ to $\mathrm{B}$ is taken to be a failure from a view of real-time systems.

In the field of computer communications, there are two categories of real-time systems. One is for hard real-time systems, and the other is for soft ones. By hard real-time systems, we mean that the time constraint, more precisely, the predetermined time delay, has to be assured. Otherwise, the communication is regarded as a failure $([4,5,10,11]$, Buttazzo [12], Raha et al. [13], Malcolm and Zhao [14], Malcolm et al. [15], Budka et al. [16], and Liem and Mendiratta [17]). By soft real-time systems, on the other side, we imply that the predetermined time constraint may be statistically violated with a predetermined probability ([10], Zhao and Chong [18], and Wang et al. [19]).

Recall that the time constraint mentioned above is the message delay suffering from $S$ from A to B (Sandmann [20], Rodríguez-Pérez et al. [21], Anjum et al [22], Papastergiou et al. [23], Panshenskov and Vakhitov [24], Kumar et al. [25], Ferrandiz et al. [26], Pin et al. [27], Florens et al. [28], Lenzini et al. [29], and Tu et al. [30]). More precisely, in the case of the Internet, this term specifically means the delay of data packets. Unless otherwise stated, this paper uses the term packet delay or delay for short. 
While we mentioned above that delay serves as a key parameter in the aspect of traffic passing through servers in the field of computer networks, one may say that the delay denoted by $d$ is actually queuing time denoted by $t_{q}$ in terms of queuing system as illustrated in Figure 1. Queuing theory may appear complex mathematically. From the point of view of applications, however, it may be quite easy to do the performance analysis of a queuing system with the basic knowledge of statistical means and standard deviations together with a pen and a piece of paper or with a few lines of code of simple computer program (Cooper [31], Reich [32], Kendall [33], Luchak [34], Little [35], Whitt [36, 37], and Li et al. [38]). Indeed, we said so if arrival traffic $X(t)$ is Markovian as those discussed in [33-38], Jagerman [39], Doshi [40], McKenna and Mitra [41], Li and Chen [42], Brandão and Nova [43], Reiman and Simon [44], Ancker Jr. and Gafarian $[45,46]$, Daley [47], and Casale et al. [48].

Note that traffic of the Markovian type implies that it is light tailed. By light tail, we mean that its autocorrelation function (ACF) is exponentially decayed and so are its power spectrum density (PSD) function and probability density function (PDF) (Li and Zhao [49, 50], Li [51]). Nevertheless, traffic is heavy tailed (Loiseau et al. [52], Hernández-Campos et al. [53], Resnick [54], Takayasu et al. [55], Willinger et al. [56], Leland et al. [57], Paxson and Floyd [58], Willinger and Paxson [59], and Beran et al. [60]), which implies that the ACF of traffic is hyperbolically decayed, that is, slowly decayed (Tsybakov and Georganas [61]). To be precise, the ACF of traffic decays slowly such that it is nonintegrable, which implies long memory or long-range dependence (LRD) (Csabai [62], Adas [63], Terdik and Gyires [64], Callado et al. [65], Owczarczuk [66], Scherrer et al. [67], Devetsikiotis and da Fonseca [68], Smith [69], Tadaki [70], Erramilli et al. [71], Karasaridis and Hatzinakos [72], Stathis and Maglaris [73], López-Ardao et al. [74], and Beran [75]). The LRD of traffic may be so strong that the variance of traffic may not exist or may be infinite ([54], Willinger et al. [76], Resnick et al. [77], López-Oliveros and Resnick [78], D’Auria and Resnick [79], and Fishman and Adan [80]). Consequently, conventional queuing theory may stop being used for analyzing queuing time or delay when arrival traffic is fractal with heavy tails or LRD such that it is of infinite variance.

Possible applications of conventional queuing theory to delay analysis are in the case of fractal traffic models with finite variance, such as fractional Brownian motion $(\mathrm{fBm})$, fractional Gaussian noise (fGn); see, for example, Norros [81], Jin and Min [82], Iftikhar et al. [83], Dahl and Willemain [84], Chevalier and Wein [85], Ou and Wein [86], Wein [87, 88], Harrison and Wein [89], Murata et al. [90], Boxma and Cohen [91], Haddad and Mazumdar [92], Ghosh and Weerasinghe [93], Duncan et al. [94], Li and Zhao [95], and Yue et al. [96]. However, overlarge buffer size may be required even when arrival fractal traffic is of finite variance (Albin and Samorodnitsky [97], Massoulie and Simonian [98], Heath et al. [99], Simonian and Guibert [100], Tsybakov and Georganas $[101,102]$, Willinger et al. [103], Kozachenko et al. [104], Carpio [105], Juneja [106], Shah and Wischik [107], and Vieira et al. [108]). The required buffer size may be so large that the value of the delay time obtained with conventional queuing theory may be impractically large for real-time systems.

The previous discussions imply that the key reason that makes the conventional queuing theory very difficult, if not impossible, to be used in the delay analysis of communication systems with fractal arrivals is the fractal properties of traffic, namely, self-similarity and LRD. Thus, fractal arrival traffic substantially challenges queuing theory of real-time systems.

As known, performance analysis of conventional queuing systems has to assume that statistical means and variances of arrival traffic exist (Cooper et al. [31-47], Pitts and Schormans [109], Stalling [110, 111], and Gibson [112]). However, generally speaking, it is inappropriate to assume that the variance of fractal traffic exists ([76-78], Li and Zhao [113], and Doukhan et al. [114]). Thus, new methodology that does not rely on statistical means and variances of arrival traffic is desired in the field of computer communication networks and real-time systems in particular.

Note that variance analysis of random functions or time series plays a key role in statistics (Bendat and Piersol [115], Gelman [116], Freedman [117], Sheskin [118], Meyer [119], Lindgren and McElrath [120], and Fuller [121]) as well as conventional queuing theory [31-47], which is actually a branch of statistics (Papoulis [122], Bhat [123]). Therefore, one may see how it is significant for us to turn away from variance analysis of arrival traffic and queuing systems to treat delay analysis of fractal traffic passing through servers. Network calculus may be a promising theory to deal with delay analysis of queuing systems, irrelevant to means and variances of arrival traffic, exhibiting remarkable advances in the aspect of queuing theory.

There are two categories with respect to the theory of network calculus. One is for deterministic delay analysis of queuing systems (Le Boudec and Thiran [124], Firoiu et al. [125], Le Boudec [126], and Cruz [127]). The other is stochastic network calculus (Jiang and Liu [128], Wang et al. [129], Burchard et al. [130], Ciucu et al. [131], and Li and Knightly [132]). We should keep in mind that the theory of stochastic network calculus substantially differs from conventional queuing theory in methodology because it follows the criterion of being irrelevant to means and variances of arrival traffic.

This paper aims at presenting novel computation methods of delay of fractal traffic passing through servers without relating to the concepts of means and variances of arrival traffic.

The rest of the paper is organized as follows. We will give the brief of fractal traffic in Section 2. In Section 3, we will exhibit the result for the delay analysis of deterministic queuing theory. Section 4 presents our delay analysis of fractal traffic passing through servers. Finally, Section 5 concludes the paper.

\section{Brief of Fractal Traffic}

Denote by $x\left(t_{i}\right)$ the arrival traffic time series (traffic for short), where $t_{i}$ is the timestamp of the $i$ th packet, where $i$ is a natural 


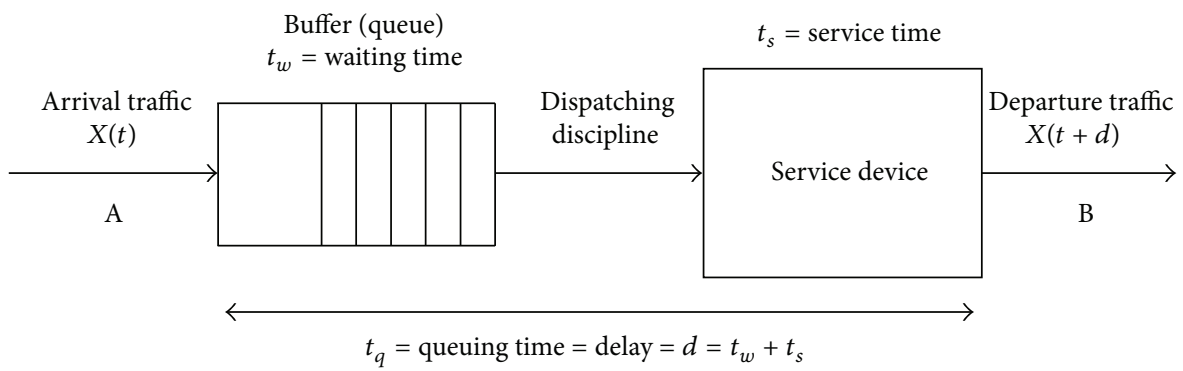

FIGURE 1: Queuing system for single server.

number (Li et al. [133]). Then, $x\left(t_{i}\right)$ implies the data size of the $i$ th packet. Since statistics of $x\left(t_{i}\right)$ is consistent with that of $x(i)$, we use $x(i)$ to indicate traffic for simplicity.

2.1. Non-Markovian Property and LRD. Denote by $r_{x x}(k)=$ $E[x(i) x(i+k)]$ the ACF of $x(i)$, where $k$ is the time lag. The ACF $r_{x x}(k)$ indicates how the size of the $i$ th packet correlates to that of another packet $(i+k)$ apart. If an ACF $R(k)$ is exponentially decayed, $R(k)$ may be neglected even for small $k$. For instance, suppose the ACF of a time series $B(i)$ that follows the Poisson distribution. It is given by (Bendat and Piersol [115])

$$
R_{B B}(k)=\exp (-2 \lambda|k|) \quad(\lambda>0) .
$$

Then, in the case of $\lambda=1$, we have

$$
R_{B B}(1) \approx 0.135 ; \quad R_{B B}(2) \approx 0.018 .
$$

Equation (2) implies that $R_{B B}(1)$ can be neglected in engineering because $x(i)$ is almost orthogonal to $x(i+1)$, letting along $R_{B B}(k)$ for $k>1$. Therefore, $R_{B B}(k) \approx 0$ for $k>0$. That means that $B(i)$ is memoryless. Accordingly, it is Markovian ([121, 122], Bunin [134], and Benes [135]). However, traffic $x(i)$ is non-Markovian, which is a property that distinguishes it from conventional time series, because $r_{x x}(k)$ is hyperbolically decayed in the form

$$
r_{x x}(k) \sim k^{-\beta}, \quad 0<\beta<1, k \longrightarrow \infty .
$$

The above implies that

$$
\sum_{0}^{\infty} r_{x x}(k)=\infty
$$

Thus, $x(i)$ is LRD or of long memory. Consequently, it is nonMarkovian (Yulmetyev et al. [136], Asgari et al. [137], van Kampen [138], Mura et al. [139], and Luczka [140]).

2.2. Property of $1 / f$ Noise. Let $S_{x x}(\omega)$ be the PSD of $x(i)$, where $\omega$ is angular frequency. According to the Wiener theorem, which is also known as the Wiener-Khintchine theorem and sometimes as the Khinchin-Kolmogorov theorem (Robinson [141], Wiener [142, 143], Khintchine [144], and Yaglom [145]), $S_{x x}(\omega)$ is the Fourier transform of $r_{x x}(k)$. Since

$$
\sum_{0}^{\infty} r_{x x}(k)=\left.S_{x x}(\omega)\right|_{\omega=0}=\infty,
$$

it is easy to infer that $S_{x x}(\omega)$ is in the form

$$
S_{x x}(\omega) \sim \frac{1}{\omega} .
$$

Therefore, $x(i)$ follows $1 / f$ noise (Mandelbrot [146, 147], Ruseckas et al. [148], Lenoir [149], Aquino et al. [150], Amir et al. [151], Carlini et al. [152, 153], Beran [154, 155], Lim and Teo [156], Eab and Lim [157], Muniandy and Lim [158], Muniandy et al. [159], Muniandy and Stanslas [160], Pinchas [161, 162], Wang and Yan [163], Bakhoum and Toma [164, 165], Yang et al. [166], Wang [167], Wornell [168], Barnes and Allan [169], Kasdin [170], and Corsini and Saletti [171]).

2.3. Self-Similarity. Traffic $x(i)$ approximately satisfies the definition of self-similarity given by

$$
x(a i) \equiv a^{H} x(i), \quad a>0,
$$

where $\equiv$ denotes equality in the sense of probability distribution and $0<H<1$ stands for the Hurst parameter [58, 61]. In general, $H$ varies with time. Hence, traffic has the property of multifractals ([108], Vieira et al. [172], Vieira and Lee [173], Masugi and Takuma [174], Masugi [175], Veitch et al. [176], Salvador et al. [177], Nogueira et al. [178], Krishna et al. [179], Feldmann et al. [180], Ayache at al. [181], Ayache [182], Liao et al. [183], Liao [184], Carbone et al. [185, 186], Stanley and Meakin [187], Yang et al. [188], Song and Shang [189], Shang et al. [190], Kantelhardt et al. [191], Ostrowsky et al. [192], Sastry et al. [193], and Min et al. [194]).

2.4. The Hurst Parameter and Fractal Dimension. Expressing $\beta$ in (3) by $H$ yields

$$
\beta=2-2 H
$$

The parameter $\beta$ is the index of LRD, and $H$ is the measure of LRD ([52, 60, 75, 76, 154], Roughan et al. [195], Abry et al. [196], and Hall and Hart [197]). In the fields, people usually use $H$ instead of $\beta$ to characterize LRD of time series for dedicating the famous hydrologist Hurst [198].

We consider the local behavior of traffic $x(i)$ using its ACF $r_{x x}(k)$. For $k \rightarrow 0$, if $r_{x x}(k)$ is sufficiently smooth on $(0, \infty)$ and if

$$
\left[r_{x x}(0)-r_{x x}(k)\right] \sim c_{1}|k|^{\alpha}, \quad 0<\alpha \leq 2,
$$


where $c_{1}$ is a constant and $\alpha$ is the fractal index of $x(i)$ (Adler [199], Chan et al. [200], Davies and Hall [201], Constantine and Hall [202], Hall and Roy [203], Kent and Wood [204], Gneiting and Schlather [205], Gneiting [206], and Lim and Teo [207]), then the fractal dimension, denoted by $D_{f}$, of $x(i)$ is given by

$$
D_{f}=2-\frac{\alpha}{2}
$$

Under the constraint of $0<\alpha \leq 2$, one has

$$
1 \leq D_{f}<2
$$

2.5. Power Laws and Heavy Tails. Taqqu's law says that the PDF of a random function $x(t)$ is in the form of a power function if it is LRD (Loiseau et al. [52], Doukhan et al. [114], Abry et al. [196], and Samorodnitsky and Taqqu [208]). Therefore, the PDF, ACF, and PSD of traffic are all in the form of power functions as can be seen from (3) and (6). When the PDF of a random function follows power laws, one says that it is heavy tailed (Adler et al. [209], Podobnik et al. [210, 211], Chen et al. [212], Xu et al. [213], Buraczewski et al. [214], Kulik and Soulier [215], Pisarenko and Rodkin [216], Resnick [217], Stanley [218], Bowers et al. [219], Eliazar and Klafter [220], Jakšić [221], Bansal et al. [222], Milojević [223], and Pareto [224]).

Denote by $p(x)$ the PDF of $x(t)$. Then, the tail of $p(x)$ may be so heavy that its mean and variance, expressed, respectively, by (12) and (13), may not exist:

$$
\begin{gathered}
E[x(t)]=\int_{-\infty}^{\infty} x p(x) d x, \\
\operatorname{Var}(x)=\int_{-\infty}^{\infty}(x-\mu)^{2} p(x) d x .
\end{gathered}
$$

2.6. Remarks. Previous discussions imply the following remarks.

Remark 1. Traffic follows power laws.

Remark 2. It is LRD.

Remark 3. It is approximately self-similar.

Remark 4. It is a type of $1 / f$ noise.

Remark 5. It is heavy tailed.

Remark 6. LRD is a global property of traffic, which is measured by $H$.

Remark 7. Fractal dimension $D_{f}$ characterizes the local selfsimilarity or local roughness or local smoothness of traffic.

In general, we do not talk about means and variances of traffic. Instead, we are interested in other two, namely, local self-similarity and LRD in the theory of fractal traffic.

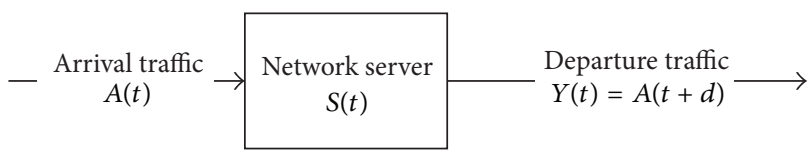

Figure 2: Traffic passing through single server.

\section{Delay of Deterministic Queuing Systems}

Network calculus may be applied to the delay analysis with respect to quality of service (QoS) in computer communication networks ([124-132], Cruz [225]). The issue of traffic passing through a server with respect to traffic delay can be described by Figure 2. The essential questions about it are stated as follows.

Question 1: how to model arrival traffic $A(t)$ towards assuring a predetermined delay, which is denoted by $D$, such that $d \leq D$ ?

Question 2: how to design a service scheme, which is denoted by $S(t)$, towards assuring a predetermined delay $D$, such that $d \leq D$ ?

Question 3: in order to guarantee the predetermined delay when $A(t)$ passes through $S(t)$, what is the operation among $A(t), Y(t)$, and $S(t)$ such that $d \leq D$ ?

The answer to question 1 is about traffic modeling. The one to question 2 is about system modeling. That to the third is the relationship among the arrival $A(t)$, the server $S(t)$, and the departure traffic $Y(t)=A(t+d)$. Three answers constitute the basic of network calculus described in [124-127, 225].

3.1. Deterministic Envelope of Traffic. In order to assure a predetermined delay $D$ such that $d \leq D$, one may utilize an envelope, which is denoted by $A(t)$, of arrival traffic $x(t)$. There are two categories of envelopes of random functions. One is in the sense of statistical envelopes, and the other is in the sense of deterministic ones.

The literature regarding statistical envelopes of lighttailed random functions is rich, as they are needed in many fields of sciences and technologies, ranging from electronics engineering to ocean one; see, for example, Rice [226, 227], Veltcheva et al. [228], Fang and Xie [229], Tayfun and Lo [230], Ochi and Sahinoglou [231, 232], Longuet-Higgins [233], Nigam [234], and Yang [235], just mentioning a few. Nonetheless, they cannot be taken as candidates of traffic envelopes because means and variances are essential to them [226-235].

In the society of computer science, people are interested in a type of envelopes of traffic, called bounding models of traffic (Michiel and Laevens [236]). Considering that arrival traffic has the property of $x(t) \geq 0$ (Li and Zhao [237]), following Cruz [127], and supposing that $x(t)$ is continuous for $t \geq 0$, a possible envelope in the time interval $[0, t]$ may be given by the inequality in the form

$$
A(t)=\int_{0}^{t} x(t) d t \leq \sigma+\rho t
$$

There are two parameters in the above expression. One is $\sigma$ that characterizes the local property of $A(t)$ called the 
burtiness in the field of computer networks ([124-127], [225], McDysan [238], Kouvatsos et al. [239], and Anantharam and Konstantopoulos $[240,241])$. The other is $\rho$ that captures the property of long-term rate of $A(t)$, citing two nice survey papers by Mao and Panwar [242] and Fidler [243], respectively, about (14).

As a matter of fact, on one hand, we have

$$
\lim _{t \rightarrow 0} A(t)=\lim _{t \rightarrow 0} \int_{0}^{t} x(t) d t \leq \sigma .
$$

Thus, $\sigma$ characterizes the burtiness of $A(t)$. On the other hand, one has

$$
\lim _{t \rightarrow \infty} \frac{A(t)}{t}=\lim _{t \rightarrow \infty} \frac{\int_{0}^{t} x(t) d t}{t} \leq \rho .
$$

Therefore, $\rho$ represents the long-term rate of $A(t)$. This model of traffic is denoted by

$$
A(t) \sim(\sigma, \rho),
$$

with the special term "Leaky Bucket" [124-127, 225, 238, 242, 243].

The deterministic envelop of traffic, namely, $A(t)$, has the properties remarked as follows.

Remark 8. $A(t)$ is increasing in the wide sense, implying that $A\left(t_{2}\right) \geq A\left(t_{1}\right)$ if $t_{2} \geq t_{1}$.

Remark 9. $A(t)$ expressed by (14) is irrelevant of statistics of $x(t)$. Consequently, we do not need the concepts of statistical means and variances for modeling traffic $x(t)$ by using $(\sigma, \rho)$.

Remark 10. Remark 9 is consistent in philosophy with fractal models of traffic.

3.2. Service Curves. Denote a service curve of a server by $S(t)$; see Figure 2. It represents a scheme of the server to allocate enough resources, such as bandwidth, to arrival traffic $A(t)$ such that the delay $d$ does not exceed the predetermined $D$. Mathematically, $S(t)$ has the same properties of $A(t)$ as described in Remarks 8-10. Thus, a function $S(t) \geq A(t)$ may be a candidate of service curve (Yin and Poo [244], Pyun et al. [245], Khanjari et al. [246], Chu et al. [247], Fulton and Li [248], Li and Hwang [249], Lau and Li [250], Li and Pruneski [251], Jamin et al. [252], Wu et al. [253], Chen et al. [254], Agrawal et al. [255], Feng et al. [256], Raha et al. [257], and Zhao and Chen [258]). Skills behind the idea of service curves appear simple, but it is significant in the development of linearizing nonlinear systems in general (Houssin et al. [259], Okumura et al. [260], and Shinzawa [261]) and queuing theory in particular [124].

3.3. Relationship among Arrival $A(t)$, Service $S(t)$, and Departure $A(t+d)$. As previously mentioned, $S(t)$ has the same properties as those of $A(t)$. Thus, we denote by $\mathbb{S}$ the set of increasing functions in the wide sense. That is, $A(t), S(t) \in \mathbb{S}$.

Let $X_{1}(t), X_{2}(t) \in \mathbb{S}$. Then, the operation expressed by (18) is called min-plus convolution [126, 262]

$$
X_{1}(t) \otimes X_{2}(t)=\inf _{0 \leq u \leq t}\left\{X_{1}(u)+X_{2}(t-u)\right\}
$$

With the tool of min-plus convolution, referring to [124-127], one has the relationship among $A(t), S(t)$, and $A(t+d)$ given by

$$
A(t) \otimes S(t) \leq Y(t)=A(t+d)
$$

3.4. Delay Computation of Single Server. The reports regarding delay computation are rich; see, for example, [124-132, 225, 244-258, 262], Raha et al. [263, 264], Ng et al. [265], Jia et al [266, 267], Amigo et al. [268], Lenzini et al. [269], Boggia et al. [270], Karam and Tobagi [271], Fukś et al. [272], Wrege et al. [273], Liebeherr et al. [274], and Golestani [275]. In this research, we present a novel way of delay computation, which is stated below.

Theorem 11. Denote by $Y_{A S}(t)=A(t) \otimes S(t)$. Then, the delay $d(t)$ that $A(t)$ suffers from $S(t)$ at time $t$ is given by

$$
d(t) \geq \frac{Y_{A S}(t)-\sigma-\rho t}{\rho} .
$$

Proof. According to (14) and (19), we have

$$
Y_{A S}(t) \leq A(t+d(t)) \leq \sigma+\rho(t+d(t)) .
$$

Thus,

$$
Y_{A S}(t) \leq \sigma+\rho(t+d(t)) .
$$

Solving $d(t)$ from the above yields (20). Thus, the theorem results.

3.5. Guaranteed Delay of Single Server. Suppose that $D$ is the predetermined deadline of delay. Then, the constraint of guaranteed delay is expressed by

$$
d(t) \leq D \quad(t>0) .
$$

In order to achieve (23), we let

$$
\frac{Y_{A S}(t)-\sigma-\rho t}{\rho} \leq d(t) \leq D .
$$

Note that $Y_{A S}(t)=A(t) \otimes S(t)$. Therefore, we may design either proper $S(t)$ or $A(t)$ or both such that (24) is satisfied. For given $A(t)$, the following theorem gives the constraint of $S(t)$ to assure (24).

Theorem 12. Denote the inverse of $\otimes$ by $\oplus$. Let $D$ be a given deadline of delay. Then, (24) is satisfied if

$$
S(t) \geq A(t) \oplus[\rho(D-t)-\sigma] .
$$

Proof. Let (24) be satisfied. Then, we have

$$
\frac{Y_{A S}(t)-\sigma-\rho t}{\rho} \leq D .
$$

Changing the sign on the left side in the above expression produces

$$
\frac{\sigma+\rho t-Y_{A S}(t)}{\rho} \geq D .
$$




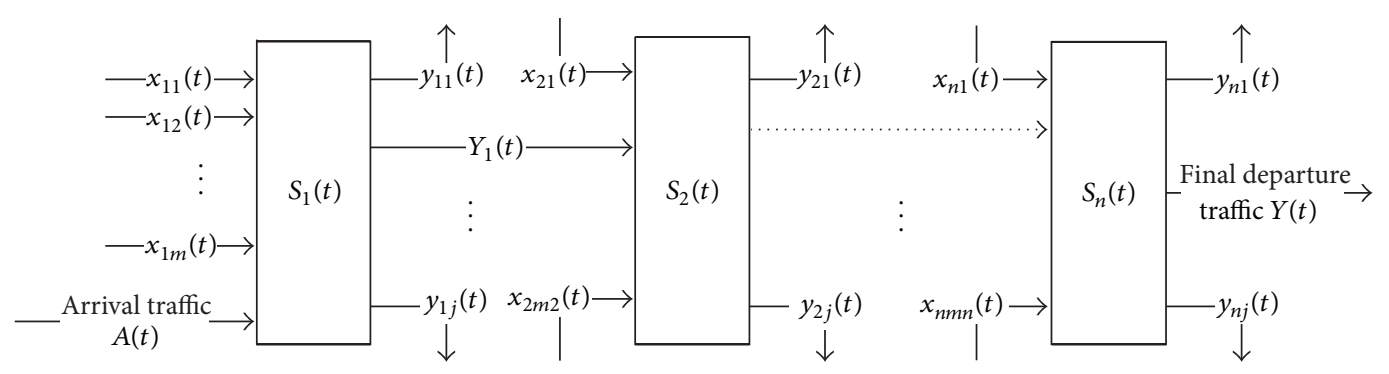

Figure 3: Arrival traffic $A(t)$ passing through $n$ servers in series.

Therefore, one has

$$
Y_{A S}(t)=A(t) \otimes S(t) \geq \rho(D-t)-\sigma .
$$

From the above, using the inverse of min-plus convolution, we have $S(t) \geq A(t) \oplus[\rho(D-t)-\sigma]$. This completes the proof.

3.6. End-to-End Delay in Tandem Network. Consider arrival traffic $A(t)$ that passes through $n$ servers in series as indicated in Figure 3. In practice, there are a number of arrival traffic that concurrently join each server at its input port and there are some traffic that may leave at the output port of that server (Coulouris et al. [276]). Either the number of traffic joining a server or leaving it is uncertain. For instance, for the first server that is denoted by $S_{1}(t)$, there are $m+1$ arrival traffic and $j+1$ departure ones at time $t$. We are only interested in the arrival denoted by $A(t)$ and the departure denoted by $Y(t)$.

One way to find the end-to-end delay of $A(t)$ passing through $n$ servers in series is to find delay $d_{i}(t)$ that $A(t)$ suffers from the $i$ th server using Theorem 11 with the constraint stated in Theorem 12. Then, the end-to-end delay at time $t$ is given by

$$
d(t)=\sum_{i=1}^{n} d_{i}(t)
$$

Denote $S(t)$ the service curve of $n$ servers in series. Then [124],

$$
S(t)=S_{1}(t) \otimes S_{2}(t) \otimes \cdots \otimes S_{n}(t)
$$

Therefore, when $S(t)$ is designed following Theorem 12 and it is decomposed into $n$ servers in series, $(23)$ is guaranteed.

The discussions in the previous subsections produce the following remarks.

Remark 13. The above delay analysis and its computations do not need any information of the statistics of arrival traffic $A(t)$.

Remark 14 . The delay can be deterministically guaranteed. Hence, the deterministic queuing systems as Le Boudec and Thiran stated in [124].

The advantage described by Remarks 13 and 14 is at cost that more resources are required (Zhao [277], Davaril et al.
[278]). In order to reduce the resource requirements that deterministic queuing analysis demands, stochastic network calculus is considered by computer scientists $([19,95,128,130$, 131, 243], Jiang et al. [279], Starobinski and Sidi [280], Ng et al. [281], Borst et al. [282], Liu et al. [283], Li et al. [284], Jiang [285], and Baccarelli et al. [286]). In what follows, we present a novel method of stochastic calculus for computing delay of fractal traffic passing through servers.

\section{Novel Delay Analysis of Fractal Traffic Passing through Servers}

We previously reported our bound of arrival traffic by taking into account its fractal dimension $D_{f}$ and the Hurst parameter $H$ [287]. It is in the form

$$
A(t)=\int_{0}^{t} x(u) d u \leq r^{2 D_{f}-5} \sigma+a^{-H} \rho t,
$$

where $r>0, a>0$. Applying (31) to Theorem 11 immediately yields a novel delay computation as stated below.

Theorem 15. Denote by $Y_{A S}(t)=A(t) \otimes S(t)$. Then, the delay $d(t)$, which $A(t)$ suffers from $S(t)$ at time $t$, is given by

$$
d(t) \geq \frac{Y_{A S}(t)-r^{2 D_{f}-5} \sigma-a^{-H} \rho t}{a^{-H} \rho} .
$$

Proof. According to (14) and (19), we obtain

$$
Y_{A S}(t) \leq A(t+d(t)) \leq r^{2 D_{f}-5} \sigma+a^{-H} \rho(t+d(t)) .
$$

Solving $d(t)$ from the above yields (32), which completes the proof.

Remark 16. The bandwidth regarding $d(t)$ expressed by (34) may be generally less than that expressed by (21).

Remark 16 is true because we take into account two parameters of fractal traffic, namely, fractal dimension and the Hurst parameter. As a matter of fact,

$$
\begin{aligned}
(\sigma+\rho t) & -\left(r^{2 D_{f}-5} \sigma+a^{-H} \rho t\right) \\
& =\left(1-r^{2 D_{f}-5}\right) \sigma+\left(1-a^{-H}\right) \rho \geq 0 .
\end{aligned}
$$

The above expression implies that, for a given $d(t)$, the bandwidth required based on Theorem 15 is less than that based on Theorem 11. 
Remark 17. Theorem 15 does not relate to statistical means and variances of arrival traffic.

Note that (31) represents a statistical bound of $A(t)$ because $D_{f}$ is a fractal parameter [199-207] and so is $H$ [198, 205-207]. $D_{f}$ expressed by (10) is with probability one and so is $H$ expressed by (8).

We previously mentioned several times that we are studying queuing systems irrelevant to statistical means and variances of arrival traffic because variances and or means of traffic may not exist $[54,76-80]$. A common case that means and variances do not exist is for random functions that follow the Cauchy distribution (G. A. Korn and T. M. Korn [288], Rice [289], and Meyer [290]). Two papers by Field et al. [291, 292] utilized the Cauchy distribution for modeling traffic. A concise explanation of random functions without mean and variance is given by Bassingthwaighte [293]. The point, namely, irrelevant to statistical means and variances of arrival traffic, makes the queuing theory based on network calculus substantially differ from the conventional one. Considering large queue size based on conventional queuing theory when arrival is fractal, network calculus may yet be an attractive theory for guaranteeing queue size in a queuing system.

\section{Conclusions}

We have explained the reasons why conventional theory of queuing systems is inappropriate to be used in the delay analysis of queuing systems when arrival traffic is fractal. Then, we have given concise method of delay computation of deterministic queuing systems. Finally, we have derived the computation method of delay when arrival traffic is fractal.

\section{Acknowledgments}

This work was supported in part by the National Natural Science Foundation of China under the project Grant nos. 61272402, 61070214, and 60873264 and by the 973 Plan under the project Grant nos. 2011CB302800.

\section{References}

[1] A. S. Tanenbaum, Computer Networks, Pearson Eduction, 4th edition, 2003.

[2] J. Postel, User Datagram Protocol, IETF RFC 768, 1980, http://www.ietf.org/rfc/rfc768.txt.

[3] J. Postel, Transmission Control Protocol, IETF RFC 793, 1981, http://www.ietf.org/rfc/rfc793.txt.

[4] W. Zhao and K. Ramamritham, "Virtual time CSMA protocols for hard real-time communications," IEEE Transactions on Software Engineering, vol. 13, no. 8, pp. 938-952, 1987.

[5] W. Zhao, K. Ramamritham, and J. A. Stankovic, "Scheduling tasks with resource requirements in hard real-time systems," IEEE Transactions on Software Engineering, vol. 13, no. 5, pp. 564-577, 1987.

[6] W. Zhao and J. A. Stankovic, "Performance analysis of FCFS and improved FCFS scheduling algorithms for dynamic realtime computer systems," in Proceedings of the Real-Time Systems
Symposium (RTSS '89), pp. 156-165, Santa Monica, Calif, USA, December 1989.

[7] R. N. Mahapatra and W. Zhao, "An energy-efficient slack distribution technique for multimode distributed real-time embedded systems," IEEE Transactions on Parallel and Distributed Systems, vol. 16, no. 7, pp. 650-662, 2005.

[8] R. Rader, "Operations support systems for mission critical public safety communication networks," Bell Labs Technical Journal, vol. 16, no. 3, pp. 151-162, 2011.

[9] T. Mahmoodi, V. Friderikos, and H. Aghvami, "Using traffic asymmetry to enhance TCP performance," Computer Networks, vol. 57, no. 1, pp. 317-329, 2013.

[10] S. Natarajan and W. Zhao, "Issues in building dynamic real-time systems," IEEE Software, vol. 9, no. 5, pp. 16-21, 1992.

[11] S. Chakraborty and J. Eberspcher, Advances in Real-Time Systems, Springer, New York, NY, USA, 2012.

[12] G. C. Buttazzo, Hard Real-Time Computing Systems, Springer, New York, NY, USA, 2011.

[13] A. Raha, S. Kamat, W. Zhao, and W. Jia, "Admission control for hard real-time connections in ATM LANs," IEE Proceedings: Communications, vol. 148, no. 4, pp. 217-228, 2001.

[14] N. Malcolm and W. Zhao, "Hard real-time communication in multiple-access networks," Real-Time Systems, vol. 8, no. 1, pp. 35-77, 1995.

[15] N. Malcolm, W. Zhao, and C. Barter, "Performance comparison of guarantee communication protocols for transmission of hard real-time messages," Australian Computer Science Communications, vol. 12, no. 1, pp. 256-265, 1990.

[16] K. C. Budka, T. Chu, T. L. Doumi, W. Brouwer, P. Lamoureux, and M. E. Palamara, "Public safety mission critical voice services over LTE," Bell Labs Technical Journal, vol. 16, no. 3, pp. 133-149, 2011.

[17] M. Liem and V. B. Mendiratta, "Mission critical communication networks for railways," Bell Labs Technical Journal, vol. 16, no. 3, pp. 29-46, 2011.

[18] W. Zhao and E. Chong, "Performance evaluation of scheduling algorithms for dynamic imprecise soft real-time computer systems," Australian Computer Science Communications, vol. 11, no. 1, pp. 329-340, 1989.

[19] S. Wang, D. Xuan, R. Bettati, and W. Zhao, "Toward statistical QoS guarantees in a differentiated services network," Telecommunication Systems, vol. 43, no. 3-4, pp. 253-263, 2010.

[20] W. Sandmann, "Delays in a series of queues with correlated service times," Journal of Network and Computer Applications, vol. 35, no. 5, pp. 1415-1423, 2012.

[21] M. Rodríguez-Pérez, S. Herrería-Alonso, M. Fernández-Veiga, and C. López-García, "Common problems in delay-based congestion control algorithms: a gallery of solutions," European Transactions on Telecommunications, vol. 22, no. 4, pp. 168-179, 2011.

[22] B. Anjum, H. Perros, X. Mountrouidou, and K. Kontovasilis, "Bandwidth allocation under end-to-end percentile delay bounds," International Journal of Network Management, vol. 21, no. 6, pp. 536-547, 2011.

[23] G. Papastergiou, C. Georgiou, L. Mamatas, and V. Tsaoussidis, "A delay-oriented prioritization policy based on non-congestive queuing," International Journal of Communication Systems, vol. 24, no. 8, pp. 1065-1086, 2011.

[24] M. A. Panshenskov and A. T. Vakhitov, "General method for network providers to choose applications with guaranteed QoS," Bell Labs Technical Journal, vol. 16, no. 2, pp. 57-61, 2011. 
[25] N. Kumar, N. Chilamkurti, and J. Lee, "A novel minimum delay maximum flow multicast algorithm to construct a multicast tree in wireless mesh networks," Computers and Mathematics with Applications, vol. 63, no. 2, pp. 481-491, 2012.

[26] T. Ferrandiz, F. Frances, and C. Fraboul, "Worst-case end-toend delays evaluation for SpaceWire networks," Discrete Event Dynamic Systems, vol. 21, no. 3, pp. 339-357, 2011.

[27] F. Pin, D. Veitch, and B. Kauffmann, "Statistical estimation of delays in a multicast tree using accelerated EM," Queueing Systems, vol. 66, no. 4, pp. 369-412, 2010.

[28] C. Florens, M. Sharif, and R. J. McEliece, "Random sensory networks: a delay analysis," IEEE Transactions on Information Theory, vol. 55, no. 4, pp. 1650-1664, 2009.

[29] L. Lenzini, E. Mingozzi, and G. Stea, "A methodology for computing end-to-end delay bounds in FIFO-multiplexing tandems," Performance Evaluation, vol. 65, no. 11-12, pp. 922943, 2008.

[30] W. Q. Tu, C. J. Sreenan, and W. J. Jia, "Worst-case delay control in multigroup overlay networks," IEEE Transactions on Parallel and Distributed Systems, vol. 18, no. 10, pp. 1407-1419, 2007.

[31] R. B. Cooper, Introduction to Queueing Theory, Elsevier, New York, NY, USA, 2nd edition, 1981.

[32] E. Reich, "Waiting times when queues are in tandem," Annals of Mathematical Statistics, vol. 28, pp. 768-773, 1957.

[33] D. G. Kendall, "Some problems in the theory of queues," Journal of the Royal Statistical Society B, vol. 13, no. 2, pp. 151-185, 1951.

[34] G. Luchak, "The continuous time solution of the equations of the single channel queue with a general class of service-time distributions by the method of generating functions," Journal of the Royal Statistical Society B, vol. 20, no. 1, pp. 176-181, 1958.

[35] J. D. C. Little, "A proof for the queuing formula: $L=\lambda W$," Operations Research, vol. 9, pp. 383-387, 1961.

[36] W. Whitt, “The queueing network analyzer," The Bell System Technical Journal, vol. 62, no. 9, pp. 2779-2815, 1983.

[37] W. Whitt, "Performance of the queueing network analyzer," The Bell System Technical Journal, vol. 62, no. 9, pp. 2817-2843, 1983.

[38] M. Li, L. Longpre, and P. Vitanyi, "Power of the queue," SIAM Journal on Computing, vol. 21, no. 4, pp. 697-712, 1992.

[39] D. L. Jagerman, "Approximate mean waiting times in transient GI/G/1 queues," The Bell System Technical Journal, vol. 61, no. 8, pp. 2003-2022, 1982.

[40] B. T. Doshi, "An M/G/1 queue with a hybrid discipline," The Bell System Technical Journal, vol. 62, no. 5, pp. 1251-1271, 1983.

[41] J. McKenna and D. Mitra, "Integral representations and asymptotic expansions for closed Markovian queueing networks: normal usage," The Bell System Technical Journal, vol. 61, no. 5, pp. 661-683, 1982.

[42] J. Li and A. Chen, "The decay parameter and invariant measures for Markovian bulk-arrival queues with control at idle time," Methodology and Computing in Applied Probability, vol. 15, no. 2, pp. 467-484, 2013.

[43] R. M. Brandão and A. M. O. P. Nova, "Analysis of eventbased, single-server nonstationary simulation responses using classical time-series models," European Journal of Operational Research, vol. 218, no. 3, pp. 676-686, 2012.

[44] M. I. Reiman and B. Simon, "Open queueing systems in light traffic," Mathematics of Operations Research, vol. 14, no. 1, pp. 26-59, 1989.
[45] C. J. Ancker Jr. and A. V. Gafarian, "Some queuing problems with balking and reneging. I," Operations Research, vol. 11, no. 1, pp. 88-100, 1963.

[46] C. J. Ancker Jr. and A. V. Gafarian, "Some queuing problems with balking and reneging. II," Operations Research, vol. 11, no. 6, pp. 928-937, 1963.

[47] D. J. Daley, "Revisiting queueing output processes: a point process viewpoint," Queueing Systems, vol. 68, no. 3, pp. 395405, 2011.

[48] G. Casale, E. Z. Zhang, and E. Smirni, "Trace data characterization and fitting for Markov modeling," Performance Evaluation, vol. 67, no. 2, pp. 61-79, 2010.

[49] M. Li and W. Zhao, "On $1 / f$ noise," Mathematical Problems in Engineering, vol. 2012, Article ID 673648, 23 pages, 2012.

[50] M. Li and W. Zhao, "Visiting power laws in cyber-physical networking systems," Mathematical Problems in Engineering, vol. 2012, Article ID 302786, 13 pages, 2012.

[51] M. Li, "Fractal time series-a tutorial review," Mathematical Problems in Engineering, vol. 2010, Article ID 157264, 26 pages, 2010.

[52] P. Loiseau, P. Gonçalves, G. Dewaele, P. Borgnat, P. Abry, and P. V. B. Primet, "Investigating self-similarity and heavy-tailed distributions on a large-scale experimental facility," IEEE/ACM Transactions on Networking, vol. 18, no. 4, pp. 1261-1274, 2010.

[53] F. Hernández-Campos, J. S. Marron, G. Samorodnitsky, and F. D. Smith, "Variable heavy tails in Internet traffic," Performance Evaluation, vol. 58, no. 2-3, pp. 261-284, 2004.

[54] S. I. Resnick, "Heavy tail modeling and teletraffic data," Annals of Statistics, vol. 25, no. 5, pp. 1805-1869, 1997.

[55] M. Takayasu, H. Takayasu, and T. Sato, "Critical behaviors and $1 / f$ noise in information traffic," Physica A, vol. 233, no. 3-4, pp. 824-834, 1996.

[56] W. Willinger, M. S. Taqqu, W. E. Leland, and D. V. Wilson, "Selfsimilarity in high-speed packet traffic: analysis and modeling of ethernet traffic measurements," Statistical Science, vol. 10, no. 1, pp. 67-85, 1995.

[57] W. E. Leland, M. S. Taqqu, W. Willinger, and D. V. Wilson, "On the self-similar nature of ethernet traffic (extended version)," IEEE/ACM Transactions on Networking, vol. 2, no. 1, pp. 1-15, 1994.

[58] V. Paxson and S. Floyd, "Wide area traffic: the failure of Poisson modeling," IEEE/ACM Transactions on Networking, vol. 3, no. 3, pp. 226-244, 1995.

[59] W. Willinger and V. Paxson, "Where mathematics meets the internet," Notices of the American Mathematical Society, vol. 45, no. 8, pp. 961-970, 1998.

[60] J. Beran, R. Sherman, M. S. Taqqu, and W. Willinger, "Longrange dependence in variable-bit-rate video traffic," IEEE Transactions on Communications, vol. 43, no. 2, pp. 1566-1579, 1995.

[61] B. Tsybakov and N. D. Georganas, "Self-similar processes in communications networks," IEEE Transactions on Information Theory, vol. 44, no. 5, pp. 1713-1725, 1998.

[62] I. Csabai, " $1 / f$ noise in computer network traffic," Journal of Physics A, vol. 27, no. 12, pp. L417-L421, 1994.

[63] A. Adas, "Traffic models in broadband networks," IEEE Communications Magazine, vol. 35, no. 7, pp. 82-89, 1997.

[64] G. Terdik and T. Gyires, "Lévy flights and fractal modeling of internet traffic," IEEE/ACM Transactions on Networking, vol. 17, no. 1, pp. 120-129, 2009. 
[65] A. Callado, C. Kamienski, G. Szabó et al., "A survey on internet traffic identification," IEEE Communications Surveys and Tutorials, vol. 11, no. 3, pp. 37-52, 2009.

[66] M. Owczarczuk, "Long memory in patterns of mobile phone usage," Physica A, vol. 391, no. 4, pp. 1428-1433, 2012.

[67] A. Scherrer, N. Larrieu, P. Owezarski, P. Borgnat, and P. Abry, "Non-Gaussian and long memory statistical characterizations for Internet traffic with anomalies," IEEE Transactions on Dependable and Secure Computing, vol. 4, no. 1, pp. 56-70, 2007.

[68] M. Devetsikiotis and N. L. S. da Fonseca, "Modeling network traffic with long range dependence: characterization, visualization and tools," Computer Networks, vol. 48, no. 3, pp. 289-291, 2005.

[69] R. D. Smith, "The dynamics of internet traffic: self-similarity, self-organization, and complex phenomena," Advances in Complex Systems, vol. 14, no. 6, pp. 905-949, 2011.

[70] S. Tadaki, "Long-term power-law fluctuation in Internet traffic," Journal of the Physical Society of Japan, vol. 76, no. 4, Article ID 044001, 5 pages, 2007.

[71] A. Erramilli, M. Roughan, D. Veitch, and W. Willinger, "Selfsimilar traffic and network dynamics," Proceedings of the IEEE, vol. 90, no. 5, pp. 800-819, 2002.

[72] A. Karasaridis and D. Hatzinakos, "Network heavy traffic modeling using $\alpha$-stable self-similar processes," IEEE Transactions on Communications, vol. 49, no. 7, pp. 1203-1214, 2001.

[73] C. Stathis and B. Maglaris, "Modelling the self-similar behaviour of network traffic," Computer Networks, vol. 34, no. 1, pp. 37-47, 2000.

[74] J. C. López-Ardao, C. López-García, A. Suárez-González, M. Fernández-Veiga, and R. Rodríguez-Rubio, "On the use of selfsimilar processes in network simulation," ACM Transactions on Modeling and Computer Simulation, vol. 10, no. 2, pp. 125-151, 2000.

[75] J. Beran, "Statistical methods for data with long-range dependence," Statistical Science, vol. 7, no. 4, pp. 404-416, 1992.

[76] W. Willinger, V. Paxson, R. H. Riedi, and M. S. Taqqu, "Longrange dependence and data network traffic," in Theory and Applications of Long-Range Dependence, P. Doukhan, G. Oppenheim, and M. S. Taqqu, Eds., pp. 373-407, Birkhäuser Boston, Boston, Mass, USA, 2002.

[77] S. Resnick, G. Samorodnitsky, and F. Xue, "How misleading can sample ACFs of stable MAs be? (very!)," Annals of Applied Probability, vol. 9, no. 3, pp. 797-817, 1999.

[78] L. López-Oliveros and S. I. Resnick, "Extremal dependence analysis of network sessions," Extremes, vol. 14, no. 1, pp. 1-28, 2011.

[79] B. D’Auria and S. I. Resnick, "The influence of dependence on data network models," Advances in Applied Probability, vol. 40, no. 1, pp. 60-94, 2008.

[80] G. S. Fishman and I. J. B. F. Adan, "How heavy-tailed distributions affect simulation-generated time averages," ACM Transactions on Modeling and Computer Simulation, vol. 16, no. 2, pp. 152-173, 2006.

[81] I. Norros, "On the use of fractional Brownian motion in the theory of connectionless networks," IEEE Journal on Selected Areas in Communications, vol. 13, no. 6, pp. 953-962, 1995.

[82] X. L. Jin and G. Y. Min, "Modelling and analysis of priority queueing systems with multi-class self-similar network traffic: a novel and efficient queue-decomposition approach," IEEE Transactions on Communications, vol. 57, no. 5, pp. 1444-1452, 2009.
[83] M. Iftikhar, T. Singh, B. Landfeldt, and M. Caglar, "Multiclass $\mathrm{G} / \mathrm{M} / 1$ queueing system with self-similar input and nonpreemptive priority," Computer Communications, vol. 31, no. 5, pp. 1012-1027, 2008.

[84] T. A. Dahl and T. R. Willemain, "The effect of long-memory arrivals on queue performance," Operations Research Letters, vol. 29, no. 3, pp. 123-127, 2001.

[85] P. B. Chevalier and L. M. Wein, "Scheduling networks of queues. Heavy traffic analysis of a multistation closed network," Operations Research, vol. 41, no. 4, pp. 743-758, 1993.

[86] J. Ou and L. M. Wein, "On the improvement from scheduling a two-station queueing network in heavy traffic," Operations Research Letters, vol. 11, no. 4, pp. 225-232, 1992.

[87] L. M. Wein, "Scheduling networks of queues. Heavy traffic analysis of a multistation network with controllable inputs," Operations Research, vol. 40, no. 2, pp. S312-S334, 1992.

[88] L. M. Wein, "Scheduling networks of queues. Heavy traffic analysis of a two-station network with controllable inputs," Operations Research, vol. 38, no. 6, pp. 1065-1078, 1990.

[89] J. M. Harrison and L. M. Wein, "Scheduling networks of queues. Heavy traffic analysis of a two-station closed network," Operations Research, vol. 38, no. 6, pp. 1052-1064, 1990.

[90] M. Murata, Y. Oie, T. Suda, and H. Miyahara, "Analysis of a discrete-time single-server queue with bursty inputs for traffic control in ATM networks," IEEE Journal on Selected Areas in Communications, vol. 8, no. 3, pp. 447-458, 1990.

[91] O. J. Boxma and J. W. Cohen, "The M/G/1 queue with heavytailed service time distribution," IEEE Journal on Selected Areas in Communications, vol. 16, no. 5, pp. 749-763, 1998.

[92] J. P. Haddad and R. R. Mazumdar, "Heavy traffic approximation for the stationary distribution of stochastic fluid networks," Queueing Systems, vol. 70, no. 1, pp. 3-21, 2012.

[93] A. P. Ghosh and A. P. Weerasinghe, "Optimal buffer size and dynamic rate control for a queueing system with impatient customers in heavy traffic," Stochastic Processes and Their Applications, vol. 120, no. 11, pp. 2103-2141, 2010.

[94] T. E. Duncan, Y. Yan, and P. Yan, "Exact asymptotics for a queue with fractional Brownian input and applications in ATM networks," Journal of Applied Probability, vol. 38, no. 4, pp. 932 945, 2001.

[95] C. Li and W. Zhao, "Stochastic performance analysis of nonfeedforward networks," Telecommunication Systems, vol. 43, no. 3-4, pp. 237-252, 2010.

[96] W. Yue, H. Takagi, and Y. Takahashi, Advances in Queueing Theory and Network Applications, Springer, New York, NY, USA, 2009.

[97] J. M. P. Albin and G. Samorodnitsky, "On overload in a storage model, with a self-similar and infinitely divisible input," Annals of Applied Probability, vol. 14, no. 2, pp. 820-844, 2004.

[98] L. Massoulie and A. Simonian, "Large buffer asymptotics for the queue with fractional Brownian input," Journal of Applied Probability, vol. 36, no. 3, pp. 894-906, 1999.

[99] D. Heath, S. Resnick, and G. Samorodnitsky, "Patterns of buffer overflow in a class of queues with long memory in the input stream," Annals of Applied Probability, vol. 7, no. 4, pp. 1021-1057, 1997.

[100] A. Simonian and J. Guibert, "Large deviations approximation for fluid queues fed by a large number of on/off sources," IEEE Journal on Selected Areas in Communications, vol. 13, no. 6, pp. 1017-1027, 1995. 
[101] B. Tsybakov and N. D. Georganas, "On self-similar traffic in atm queues: definitions, overflow probability bound, and cell delay distribution," IEEE/ACM Transactions on Networking, vol. 5, no. 3, pp. 397-409, 1997.

[102] B. Tsybakov and N. D. Georganas, "Self-similar traffic and upper bounds to buffer-overflow probability in an ATM queue," Performance Evaluation, vol. 32, no. 1, pp. 57-80, 1998.

[103] W. Willinger, M. S. Taqqu, R. Sherman, and D. V. Wilson, "Selfsimilarity through high-variability: statistical analysis of Ethernet LAN traffic at the source level," IEEE/ACM Transactions on Networking, vol. 5, no. 1, pp. 71-86, 1997.

[104] Y. Kozachenko, O. Vasylyk, and T. Sottinen, "Path space large deviations of a large buffer with Gaussian input traffic," Queueing Systems, vol. 42, no. 2, pp. 113-129, 2002.

[105] K. J. E. Carpio, "Long-range dependence of stationary processes in single-server queues," Queueing Systems, vol. 55, no. 2, pp. 123-130, 2007.

[106] S. Juneja, "Estimating tail probabilities of heavy tailed distributions with asymptotically zero relative error," Queueing Systems, vol. 57, no. 2-3, pp. 115-127, 2007.

[107] D. Shah and D. Wischik, "Fluid models of congestion collapse in overloaded switched networks," Queueing Systems, vol. 69, no. 2, pp. 121-143, 2011.

[108] F. H. T. Vieira, F. G. C. Rocha, and J. A. dos Santos Jr., "Loss probability estimation and control for OFDM/TDMA wireless systems considering multifractal traffic characteristics," Computer Communications, vol. 35, no. 2, pp. 263-271, 2012.

[109] J. M. Pitts and J. A. Schormans, Introduction to IP and ATM Design and Performance: With Applications and Analysis Software, John Wiley \& Sons, New York, NY, USA, 2000.

[110] W. Stalling, Data and Computer Communications, Macmillan, New York, NY, USA, 4th edition, 1994.

[111] W. Stallings, High-Speed Networks: TCP/IP and ATM Design Principles, Prentice Hall, New York, NY, USA, 1998.

[112] J. D. Gibson, The Communications Handbook, IEEE Press, 1997.

[113] M. Li and W. Zhao, "Wild fluctuations of random functions with the Pareto distribution," Mathematical Problems in Engineering, vol. 2013, Article ID 767502, 3 pages, 2013.

[114] P. Doukhan, G. Oppenheim, and M. S. Taqqu, Theory and Applications of Long-Range Dependence, Birkhäuser, Boston, Mass, USA, 2003.

[115] J. S. Bendat and A. G. Piersol, Random Data: Analysis and Measurement Procedure, Wiley Series in Probability and Statistics, John Wiley \& Sons, Hoboken, NJ, USA, 3rd edition, 2000.

[116] A. Gelman, "Discussion paper analysis of variance-why it is more important than ever," Annals of Statistics, vol. 33, no. 1, pp. 1-53, 2005.

[117] D. A. Freedman, Statistical Models: Theory and Practice, Cambridge University Press, Cambridge, UK, 2005.

[118] D. Sheskin, Statistical Tests and Experimental Design: A Guidebook, Gardner Press, New York, NY, USA, 1984.

[119] S. L. Meyer, Data Analysis for Scientists and Engineers, John Wiley \& Sons, New York, NY, USA, 1975.

[120] B. W. Lindgren and G. W. McElrath, Introduction to Probability and Statistics, Macmillan, New York, NY, USA, 1959.

[121] W. A. Fuller, Introduction to Statistical Time Series, Wiley Series in Probability and Statistics: Probability and Statistics, John Wiley \& Sons, New York, NY, USA, 2nd edition, 1996.

[122] A. Papoulis, Probability, Random Variables, and Stochastic Processes, McGraw-Hill, New York, NY, USA, 2nd edition, 1984.
[123] U. N. Bhat, "Sixty years of queueing theory", Management Science, vol. 15, pp. 280-294, 1968/1969.

[124] J. Y. Le Boudec and P. Thiran, Network Calculus: A Theory of Deterministic Queuing Systems for the Internet, vol. 2050 of Lecture Notes in Computer Science, Springer, Berlin, Germany, 2001.

[125] V. Firoiu, J. Y. Le Boudec, D. Towsley, and Z. L. Zhang, “Theories and models for internet quality of service," Proceedings of the IEEE, vol. 90, no. 9, pp. 1565-1591, 2002.

[126] J. Y. Le Boudec, "Application of network calculus to guaranteed service networks," IEEE Transactions on Information Theory, vol. 44, no. 3, pp. 1087-1096, 1998.

[127] R. L. Cruz, "A calculus for network delay-I: network elements in isolation," IEEE Transactions on Information Theory, vol. 37, no. 1, pp. 114-131, 1991.

[128] Y. M. Jiang and Y. Liu, Stochastic Network Calculus, Springer, New York, NY, USA, 2008.

[129] S. Wang, Z. Mai, D. Xuan, and W. Zhao, "Design and implementation of QoS-provisioning system for voice over IP," IEEE Transactions on Parallel and Distributed Systems, vol. 17, no. 3, pp. 276-288, 2006.

[130] A. Burchard, J. Liebeherr, and S. D. Patek, "A min-plus calculus for end-to-end statistical service guarantees," IEEE Transactions on Information Theory, vol. 52, no. 9, pp. 4105-4114, 2006.

[131] F. Ciucu, A. Burchard, and J. Liebeherr, "Scaling properties of statistical end-to-end bounds in the network calculus," IEEE Transactions on Information Theory, vol. 52, no. 6, pp. 23002312, 2006.

[132] C. Li and E. W. Knightly, "Schedulability criterion and performance analysis of coordinated schedulers," IEEE/ACM Transactions on Networking, vol. 13, no. 2, pp. 276-287, 2005.

[133] M. Li, W. Jia, and W. Zhao, "Correlation form of timestamp increment sequences of self-similar traffic on Ethernet," Electronics Letters, vol. 36, no. 19, pp. 1668-1669, 2000.

[134] B. J. Bunin, "Rate-distortion function for Gaussian Markov processes," The Bell System Technical Journal, vol. 48, no. 9, pp. 3059-3074, 1969.

[135] V. E. Beneš, "Markov processes representing traffic in connecting networks," The Bell System Technical Journal, vol. 42, pp. 2795-2837, 1963.

[136] R. M. Yulmetyev, N. Emelyanova, P. Hänggi, F. Gafarov, and A. Prokhorov, "Long-range memory and non-Markov statistical effects in human sensorimotor coordination," Physica A, vol. 316, no. 1-4, pp. 671-687, 2002.

[137] H. Asgari, S. V. Muniandy, and C. S. Wong, "Stochastic dynamics of charge fluctuations in dusty plasma: a non-Markovian approach," Physics of Plasmas, vol. 18, no. 8, Article ID 083709, 2011.

[138] N. G. van Kampen, "Remarks on non-Markov processes," Brazilian Journal of Physics, vol. 28, no. 2, pp. 90-96, 1998.

[139] A. Mura, M. S. Taqqu, and F. Mainardi, "Non-Markovian diffusion equations and processes: analysis and simulations," Physica A, vol. 387, no. 21, pp. 5033-5064, 2008.

[140] J. Łuczka, "Non-Markovian stochastic processes," Chaos, vol. 15, no. 2, Article ID 026107, 13 pages, 2005.

[141] E. A. Robinson, "A historical perspective of spectrum estimation," Proceedings of the IEEE, vol. 70, no. 9, pp. 885-907, 1982.

[142] N. Wiener, Time Series, MIT Press, Cambridge, Mass, USA, 1964. 
[143] N. Wiener, Extrapolation, Interpolation, and Smoothing of Stationary Time Series, MIT Press, Cambridge, Mass, USA, 1949.

[144] A. Khintchine, "Korrelationstheorie der stationären stochastischen Prozesse," Mathematische Annalen, vol. 109, no. 1, pp. 604615, 1934.

[145] A. M. Yaglom, Correlation Theory of Stationary and Related Random Functions, Volume I: Basic Results, Springer Series in Statistics, Springer, New York, NY, USA, 1987.

[146] B. B. Mandelbrot, Multifractals and $1 / f$ noise, Springer, New York, NY, USA, 1998.

[147] B. B. Mandelbrot, "Some noises with $1 / f$ spectrum, a bridge between direct current and white noise," IEEE Transactions on Information Theory, vol. 13, no. 2, pp. 289-298, 1967.

[148] J. Ruseckas, B. Kaulakys, and V. Gontis, "Herding model and $1 / f$ noise," Europhysics Letters, vol. 96, no. 6, Article ID 60007, 2011.

[149] B. Lenoir, "Predicting the variance of a measurement with $1 / f$ noise," Fluctuation and Noise Letters, vol. 12, no. 1, Article ID 1350006, 6 pages, 2013.

[150] G. Aquino, M. Bologna, P. Grigolini, and B. J. West, "Beyond the death of linear response: 1/f optimal information transport," Physical Review Letters, vol. 105, no. 6, Article ID 040601, 4 pages, 2010.

[151] A. Amir, Y. Oreg, and Y. Imry, " $1 / f$ noise and slow relaxations in glasses," Annalen der Physik, vol. 18, no. 12, pp. 836-843, 2010.

[152] M. Carlini, T. Honorati, and S. Castellucci, "Photovoltaic greenhouses: comparison of optical and thermal behaviour for energy savings," Mathematical Problems in Engineering, vol. 2012, Article ID 743764, 10 pages, 2012.

[153] M. Carlini, S. Castellucci, E. Allegrini, and A. Tucci, "Downhole heat exchangers: modelling of a low-enthalpy geothermal system for district heating," Mathematical Problems in Engineering, vol. 2012, Article ID 845192, 11 pages, 2012.

[154] J. Beran, Statistics for Long-Memory Processes, vol. 61 of Monographs on Statistics and Applied Probability, Chapman \& Hall, New York, NY, USA, 1994.

[155] J. Beran, "Discussion: heavy tail modeling and teletraffic data," Annals of Statistics, vol. 25, no. 5, pp. 1852-1856, 1997.

[156] S. C. Lim and L. P. Teo, "The fractional oscillator process with two indices," Journal of Physics A, vol. 42, no. 6, Article ID 065208, 2009.

[157] C. H. Eab and S. C. Lim, "Accelerating and retarding anomalous diffusion," Journal of Physics A, vol. 45, no. 14, Article ID 145001, 17 pages, 2012.

[158] S. V. Muniandy and S. C. Lim, "Modeling of locally self-similar processes using multifractional Brownian motion of RiemannLiouville type," Physical Review E, vol. 63, no. 4, Article ID 046104, 2001.

[159] S. V. Muniandy, W. X. Chew, and C. S. Wong, "Fractional dynamics in the light scattering intensity fluctuation in dusty plasma," Physics of Plasmas, vol. 18, no. 1, Article ID 013701, 2011.

[160] S. V. Muniandy and J. Stanslas, "Modelling of chromatin morphologies in breast cancer cells undergoing apoptosis using generalized Cauchy field," Computerized Medical Imaging and Graphics, vol. 32, no. 7, pp. 631-637, 2008.

[161] M. Pinchas, "Symbol error rate as a function of the residual ISI obtained by blind adaptive equalizers for the SIMO and fractional Gaussian noise case," Mathematical Problems in Engineering, vol. 2013, Article ID 860389, 9 pages, 2013.

[162] M. Pinchas, "Residual ISI obtained by blind adaptive equalizers and fractional noise," Mathematical Problems in Engineering, vol. 2013, Article ID 972174, 11 pages, 2013.
[163] Z. Wang and L. Yan, "The S-transform of sub-fBm and an application to a class of linear sub-fractional BSDEs," Advances in Mathematical Physics, vol. 2013, Article ID 827192, 11 pages, 2013.

[164] E. G. Bakhoum and C. Toma, "Specific mathematical aspects of dynamics generated by coherence functions," Mathematical Problems in Engineering, vol. 2011, Article ID 436198, 10 pages, 2011.

[165] E. G. Bakhoum and C. Toma, "Modeling transitions in complex systems by multiplicative effect of temporal patterns extracted from signal flows," Mathematical Problems in Engineering, vol. 2012, Article ID 409856, 11 pages, 2012.

[166] J. W. Yang, Y. J. Chen, and M. Scalia, "Construction of affine invariant functions in spatial domain," Mathematical Problems in Engineering, vol. 2012, Article ID 690262, 11 pages, 2012.

[167] Y. M. Wang, "Maximum norm error estimates of ADI methods for a two-dimensional fractional subdiffusion equation," Advances in Mathematical Physics, vol. 2013, Article ID 293706, 10 pages, 2013.

[168] G. W. Wornell, "Wavelet-based representations for the $1 / f$ family of fractal processes," Proceedings of the IEEE, vol. 81, no. 10, pp. 1428-1450, 1993.

[169] J. A. Barnes and D. W. Allan, "A statistical model of flicker noise," Proceedings of the IEEE, vol. 54, no. 2, pp. 176-178, 1966.

[170] N. J. Kasdin, "Discrete simulation of colored noise and stochastic processes and $1 / f^{\alpha}$ power law noise generation," Proceedings of the IEEE, vol. 83, no. 5, pp. 802-827, 1995.

[171] G. Corsini and R. Saletti, " $1 / f^{\gamma}$ power spectrum noise sequence generator," IEEE Transactions on Instrumentation and Measurement, vol. 37, no. 4, pp. 615-619, 1988.

[172] F. H. T. Vieira, G. R. Bianchi, and L. L. Lee, "A network traffic prediction approach based on multifractal modeling," Journal of High Speed Networks, vol. 17, no. 2, pp. 83-96, 2008.

[173] F. H. T. Vieira and L. L. Lee, "Adaptive wavelet-based multifractal model applied to the effective bandwidth estimation of network traffic flows," IET Communications, vol. 3, no. 6, pp. 906-919, 2009.

[174] M. Masugi and T. Takuma, "Multi-fractal analysis of IP-network traffic for assessing time variations in scaling properties," Physica D, vol. 225, no. 2, pp. 119-126, 2007.

[175] M. Masugi, "Multi-fractal analysis of IP-network traffic based on a hierarchical clustering approach," Communications in Nonlinear Science and Numerical Simulation, vol. 12, no. 7, pp. 1316-1325, 2007.

[176] D. Veitch, N. Hohn, and P. Abry, "Multifractality in TCP/IP traffic: the case against," Computer Networks, vol. 48, no. 3, pp. 293-313, 2005.

[177] P. Salvador, A. Nogueira, and R. Valadas, "Framework based on stochastic L-Systems for modeling IP traffic with multifractal behavior," Computer Communications, vol. 27, no. 18, pp. 17991811, 2004.

[178] A. Nogueira, P. Salvador, R. Valadas, and A. Pacheco, "Modeling network traffic with multifractal behavior," Telecommunication Systems, vol. 24, no. 2-4, pp. 339-362, 2003.

[179] P. M. Krishna, V. M. Gadre, and U. B. Desai, "Modelling and control of broad band traffic using multiplicative multifractal cascades," Sadhana, vol. 27, no. 6, pp. 699-723, 2002.

[180] A. Feldmann, A. C. Gilbert, W. Willinger, and T. G. Kurtz, "The changing nature of network traffic: scaling phenomena," ACM SIGCOMM Computer Communication Review, vol. 28, no. 2, pp. 5-29, 1998. 
[181] A. Ayache, S. Cohen, and J. L. Vehel, "Covariance structure of multifractional Brownian motion, with application to long range dependence," in Proceedings of the IEEE Interntional Conference on Acoustics, Speech, and Signal Processing (ICASSP '00), vol. 6, pp. 3810-3813, June 2000.

[182] A. Ayache, "Continuous Gaussian multifractional processes with random pointwise Hölder regularity," Journal of Theoretical Probability, vol. 26, no. 1, pp. 72-93, 2013.

[183] Z. W. Liao, S. X. Hu, D. Sun, and W. F. Chen, "Enclosed laplacian operator of nonlinear anisotropic diffusion to preserve singularities and delete isolated points in image smoothing," Mathematical Problems in Engineering, vol. 2011, Article ID 749456, 15 pages, 2011.

[184] Z. W. Liao, "Regularization multi directions and multi scales anisotropic diffusion for sinogram restoration of low-dosed computed tomography," Mathematical Problems in Engineering. In press.

[185] A. Carbone, G. Castelli, and H. E. Stanley, "Analysis of clusters formed by the moving average of a long-range correlated time series," Physical Review E, vol. 69, no. 2, Article ID 026105, 4 pages, 2004.

[186] A. Carbone, G. Castelli, and H. E. Stanley, "Time-dependent Hurst exponent in financial time series," Physica A, vol. 344, no. 1-2, pp. 267-271, 2004.

[187] H. E. Stanley and P. Meakin, "Multifractal phenomena in physics and chemistry," Nature, vol. 335, no. 6189, pp. 405-409, 1988.

[188] J. W. Yang, Z. Chen, W. S. Chen, and Y. J. Chen, "Robust affine invariant descriptors," Mathematical Problems in Engineering, vol. 2011, Article ID 185303, 15 pages, 2011.

[189] J. Song and P. J. Shang, "Effect of linear and nonlinear filters on multifractal detrended cross-correlation analysis," Fractals, vol. 19, no. 4, pp. 443-453, 2011.

[190] P. J. Shang, Y. Lu, and S. Kama, "The application of Hölder exponent to traffic congestion warning," Physica A, vol. 370, no. 2, pp. 769-776, 2006.

[191] J. W. Kantelhardt, S. A. Zschiegner, E. Koscielny-Bunde, S. Havlin, A. Bunde, and H. E. Stanley, "Multifractal detrended fluctuation analysis of nonstationary time series," Physica A, vol. 316, no. 1-4, pp. 87-114, 2002.

[192] L. O. Ostrowsky, N. L. S. da Fonseca, and C. A. V. Melo, "A multiscaling traffic model for UDP streams," Simulation Modelling Practice and Theory, vol. 26, pp. 32-48, 2012.

[193] C. S. Sastry, S. Rawat, A. K. Pujari, and V. P. Gulati, "Network traffic analysis using singular value decomposition and multiscale transforms," Information Sciences, vol. 177, no. 23, pp. 5275-5291, 2007.

[194] G. Min, M. Ould-Khaoua, S. Loucif, and H. Yin, "An analytical model for hypercubes in the presence of multiple time-scale bursty traffic," Journal of Systems Architecture, vol. 52, no. 7, pp. 423-431, 2006.

[195] M. Roughan, D. Veitch, and P. Abry, "Real-time estimation of the parameters of long-range dependence," IEEE/ACM Transactions on Networking, vol. 8, no. 4, pp. 467-478, 2000.

[196] P. Abry, P. Borgnat, F. Ricciato, A. Scherrer, and D. Veitch, "Revisiting an old friend: on the observability of the relation between long range dependence and heavy tail," Telecommunication Systems, vol. 43, no. 3-4, pp. 147-165, 2010.

[197] P. Hall and J. D. Hart, "Nonparametric regression with longrange dependence," Stochastic Processes and Their Applications, vol. 36, no. 2, pp. 339-351, 1990.
[198] H. E. Hurst, "Long term storage capacity of reservoirs," Transactions of the American Society of Civil Engineers, vol. 116, pp. 770-799, 1951.

[199] R. J. Adler, The Geometry of Random Fields, John Wiley \& Sons, New York, NY, USA, 1981.

[200] G. Chan, P. Hall, and D. S. Poskitt, "Periodogram-based estimators of fractal properties," Annals of Statistics, vol. 23, no. 5, pp. 1684-1711, 1995.

[201] S. Davies and P. Hall, "Fractal analysis of surface roughness by using spatial data," Journal of the Royal Statistical Society B, vol. 61, no. 1, pp. 3-37, 1999.

[202] A. G. Constantine and P. Hall, "Characterizing surface smoothness via estimation of effective fractal dimension," Journal of the Royal Statistical Society B, vol. 56, no. 1, pp. 97-113, 1994.

[203] P. Hall and R. Roy, "On the relationship between fractal dimension and fractal index for stationary stochastic processes," The Annals of Applied Probability, vol. 4, no. 1, pp. 241-253, 1994.

[204] J. T. Kent and A. T. A. Wood, "Estimating the fractal dimension of a locally self-similar Gaussian process by using increments," Journal of the Royal Statistical Society B, vol. 59, no. 3, pp. 679$699,1997$.

[205] T. Gneiting and M. Schlather, "Stochastic models that separate fractal dimension and the hurst effect," SIAM Review, vol. 46, no. 2, pp. 269-282, 2004.

[206] T. Gneiting, "Power-law correlations, related models for longrange dependence and their simulation," Journal of Applied Probability, vol. 37, no. 4, pp. 1104-1109, 2000.

[207] S. C. Lim and L. P. Teo, "Gaussian fields and Gaussian sheets with generalized Cauchy covariance structure," Stochastic Processes and Their Applications, vol. 119, no. 4, pp. 1325-1356, 2009.

[208] G. Samorodnitsky and M. S. Taqqu, Stable Non-Gaussian Random Processes, Chapman \& Hall, New York, NY, USA, 1994.

[209] R. J. Adler, R. Feldman, and M. S. Taqqu, A Practical Guide to Heavy Tails: Statistical Techniques and Applications, Birkhäuser, Boston, Mass, USA, 1998.

[210] B. Podobnik, P. C. Ivanov, K. Biljakovic, D. Horvatic, H. E. Stanley, and I. Grosse, "Fractionally integrated process with power-law correlations in variables and magnitudes," Physical Review E, vol. 72, no. 2, Article ID 026121, 7 pages, 2005.

[211] B. Podobnik, P. C. Ivanov, V. Jazbinsek, Z. Trontelj, H. E. Stanley, and I. Grosse, "Power-law correlated processes with asymmetric distributions," Physical Review E, vol. 71, no. 2, Article ID 025104, 4 pages, 2005.

[212] Z. Chen, P. C. Ivanov, K. Hu, and H. E. Stanley, "Effect of nonstationarities on detrended fluctuation analysis," Physical Review E, vol. 65, no. 4, Article ID 041107, 15 pages, 2002.

[213] L. Xu, P. C. Ivanov, K. Hu, Z. Chen, A. Carbone, and H. E. Stanley, "Quantifying signals with power-law correlations: a comparative study of detrended fluctuation analysis and detrended moving average techniques," Physical Review E, vol. 71, no. 5, Article ID 051101, 14 pages, 2005.

[214] D. Buraczewski, E. Damek, S. Mentemeier, and M. Mirek, "Heavy tailed solutions of multivariate smoothing transforms," Stochastic Processes and Their Applications, vol. 123, no. 6, pp. 1947-1986, 2013.

[215] R. Kulik and P. Soulier, "Estimation of limiting conditional distributions for the heavy tailed long memory stochastic volatility process," Extremes, vol. 16, no. 2, pp. 203-239, 2013.

[216] V. Pisarenko and M. Rodkin, Heavy-Tailed Distributions in Disaster Analysis, Springer, New York, NY, USA, 2010. 
[217] S. I. Resnick, Heavy-Tail Phenomena: Probabilistic and Statistical Modeling, Springer Series in Operations Research and Financial Engineering, Springer, New York, NY, USA, 2007.

[218] H. E. Stanley, "Power laws and universality," Nature, vol. 378, no. 6557, p. 554, 1995.

[219] M. C. Bowers, W. W. Tung, and J. B. Gao, "On the distributions of seasonal river flows: lognormal or power law?" Water Resources Research, vol. 48, Article ID W05536, 12 pages, 2012.

[220] I. Eliazar and J. Klafter, "A probabilistic walk up power laws," Physics Reports, vol. 511, no. 3, pp. 143-175, 2012.

[221] N. Jakšić, "Power law damping parameter identification," Journal of Sound and Vibration, vol. 330, no. 24, pp. 5878-5893, 2011.

[222] A. R. Bansal, G. Gabriel, and V. P. Dimri, "Power law distribution of susceptibility and density and its relation to seismic properties: an example from the German continental deep drilling program (KTB)," Journal of Applied Geophysics, vol. 72, no. 2, pp. 123-128, 2010.

[223] S. Milojević, "Power law distributions in information science: making the case for logarithmic binning," Journal of the American Society for Information Science and Technology, vol. 61, no. 12, pp. 2417-2425, 2010.

[224] V. Pareto, "La legge della domanda," Giornale degli Economisti, vol. 10, pp. 59-68, 1895, English translation in Rivista di Politica Economica, vol. 87, pp. 691-700, 1997.

[225] R. L. Cruz, "Quality of service guarantees in virtual circuit switched networks," IEEE Journal on Selected Areas in Communications, vol. 13, no. 6, pp. 1048-1056, 1995.

[226] S. O. Rice, "Mathematical analysis of random noise, parts III and IV," The Bell System Technical Journal, vol. 24, pp. 46-156, 1945.

[227] S. O. Rice, "Mathematical analysis of random noise," The Bell System Technical Journal, vol. 23, pp. 282-332, 1944.

[228] A. Veltcheva, P. Cavaco, and C. G. Soares, "Comparison of methods for calculation of the wave envelope," Ocean Engineering, vol. 30, no. 7, pp. 937-948, 2003.

[229] Z. S. Fang and N. Xie, "An analysis of various methods for computing the envelope of a random signal," Applied Ocean Research, vol. 17, no. 1, pp. 9-19, 1995.

[230] M. A. Tayfun and J. M. Lo, "Wave envelope and related spectra," Journal of Waterway, Port, Coastal and Ocean Engineering, vol. 115, no. 4, pp. 515-533, 1989.

[231] M. K. Ochi and I. I. Sahinoglou, "Stochastic characteristics of wave groups in random seas: part 1-time duration of and number of waves in a wave group," Applied Ocean Research, vol. 11, no. 1, pp. 39-50, 1989.

[232] M. K. Ochi and I. I. Sahinoglou, "Stochastic characteristics of wave groups in random seas: part 2-Frequency of occurrence of wave groups," Applied Ocean Research, vol. 11, no. 2, pp. 8999, 1989.

[233] M. S. Longuet-Higgins, "Statistical properties of wave groups in a random sea state," Philosophical Transactions of the Royal Society of London A, vol. 312, no. 1521, pp. 219-250, 1984.

[234] N. C. Nigam, Introduction to Random Vibrations, MIT Press, Cambridge, Mass, USA, 1983.

[235] J. N. Yang, "Simulation of random envelope processes," Journal of Sound and Vibration, vol. 21, no. 1, pp. 73-85, 1972.

[236] H. Michiel and K. Laevens, "Teletraffic engineering in a broadband era," Proceedings of the IEEE, vol. 85, no. 12, pp. 2007-2033, 1997.
[237] M. Li and W. Zhao, "Asymptotic identity in min-plus algebra: a report on CPNS," Computational and Mathematical Methods in Medicine, vol. 2012, Article ID 154038, 11 pages, 2012.

[238] D. McDysan, QoS \& Traffic Management in IP \& ATM Networks, McGraw-Hill, New York, NY, USA, 2000.

[239] D. D. Kouvatsos, I. Awan, and K. Al-Begain, "Performance modelling of GPRS with bursty multiclass traffic," IEE Proceedings: Computers and Digital Techniques, vol.150, no. 2, pp. 75-85, 2003.

[240] V. Anantharam and T. Konstantopoulos, "Burst reduction properties of the leaky bucket flow control scheme in ATM networks," IEEE Transactions on Communications, vol. 42, no. 12, pp. 3085-3089, 1994.

[241] V. Anantharam and T. Konstantopoulos, "A methodology for the design of optimal traffic shapers in communication networks," IEEE Transactions on Automatic Control, vol. 44, no. 3, pp. 583-586, 1999.

[242] S. W. Mao and S. S. Panwar, "A survey of envelope processes and their applications in quality of service provisioning," IEEE Communications Surveys \& Tutorials, vol. 8, no. 3, pp. 2-20, 2006.

[243] M. Fidler, "A survey of deterministic and stochastic service curve models in the network calculus," IEEE Communications Surveys and Tutorials, vol. 12, no. 1, pp. 59-86, 2010.

[244] Y. Yin and G. S. Poo, "End-to-end QoS guarantees for a network based on Latency-Rate Max-Min service curve," Computer Communications, vol. 29, no. 18, pp. 3833-3843, 2006.

[245] K. Pyun, J. Song, and H. K. Lee, "The service curve service discipline for the rate-controlled EDF service discipline in variable-sized packet networks," Computer Communications, vol. 29, no. 18, pp. 3886-3899, 2006.

[246] S. A. Khanjari, B. Arafeh, K. Day, and N. Alzeidi, "Bandwidth borrowing-based QoS approach for adaptive call admission control in multiclass traffic wireless cellular networks," International Journal of Communication Systems, vol. 26, no. 7, pp. 811831, 2013.

[247] W. Chu, X. Guan, Z. Cai, and L. Gao, "Real-time volume control for interactive network traffic replay," Computer Networks, vol. 57, no. 7, pp. 1611-1629, 2013.

[248] C. A. Fulton and S. Q. Li, "Delay jitter first-order and secondorder statistical functions of general traffic on high-speed multimedia networks," IEEE/ACM Transactions on Networking, vol. 6, no. 2, pp. 150-163, 1998.

[249] S. Q. Li and C. L. Hwang, "On the convergence of traffic measurement and queueing analysis: a statistical-matching and queueing (SMAQ) tool," IEEE/ACM Transactions on Networking, vol. 5, no. 1, pp. 95-110, 1997.

[250] W. C. Lau and S. Q. Li, "Statistical multiplexing and buffer sharing in multimedia high-speed networks: a frequencydomain perspective," IEEE/ACM Transactions on Networking, vol. 5, no. 3, pp. 382-396, 1997.

[251] S. Q. Li and J. D. Pruneski, "The linearity of low frequency traffic flow: an intrinsic I/O property in queueing systems," IEEE/ACM Transactions on Networking, vol. 5, no. 3, pp. 429-443, 1997.

[252] S. Jamin, P. B. Danzig, S. J. Shenker, and L. X. Zhang, "A measurement-based admission control algorithm for integrated service packet networks," IEEE/ACM Transactions on Networking, vol. 5, no. 1, pp. 56-70, 1997.

[253] J. J. Wu, J. C. Liu, and W. Zhao, "A general framework for parameterized schedulability bound analysis of real-time 
systems," IEEE Transactions on Computers, vol. 59, no. 6, pp. 776-783, 2010.

[254] B. Chen, S. Kamat, and W. Zhao, "Fault-tolerant, real-time communication in FDDI-based networks," Computer, vol. 30, no. 4, pp. 83-90, 1997.

[255] G. Agrawal, B. Chen, and S. Davari, "Guaranteeing synchronous message deadlines with the timed token medium access control protocol," IEEE Transactions on Computers, vol. 43, no. 3, pp. 327-339, 1994.

[256] F. Feng, W. Zhao, and A. Kumar, "Bounding applicationto-application delays for multimedia traffic in FDDI-based communication systems," in Multimedia Computing and Networking, vol. 2667 of Proceedings of the SPIE, pp. 174-185, San Jose, Calif, USA, January 1996.

[257] A. Raha, S. Kamat, and W. Zhao, "Admission control for hard real-time connections in ATM LANs," in Proceedings of the 15th Annual Joint Conference of the IEEE Computer and Communications Societies (INFOCOM '96), vol. 1, pp. 180-188, San Francisco, Calif, USA, March 1996.

[258] W. Zhao and B. Chen, "On guaranteeing message deadlines in ATM-based heterogeneous networks," in Proceedings of the International Conference of Computing Science, pp. 186-195, Minneapolis, Minn, USA, October 1996.

[259] L. Houssin, S. Lahaye, and J. L. Boimond, "Control of (max, +)-linear systems minimizing delays," Discrete Event Dynamic Systems, vol. 23, no. 3, pp. 261-276, 2013.

[260] T. Okumura, J. Matsukidaira, and D. Takahashi, "Max-minplus expressions for one-dimensional particle cellular automata obtained from a fundamental diagram," Journal of Physics A, vol. 46, no. 29, Article ID 295101, 2013.

[261] N. Shinzawa, "Elimination of variables and the determinant of the max plus algebra," Journal of Physics A, vol. 45, no. 10, Article ID 105202, 27 pages, 2012.

[262] M. Li and W. Zhao, Analysis of Min-Plus Algebra, Nova Science Publishers, 2011.

[263] A. Raha, S. Kamat, and W. Zhao, "Guaranteeing end-to-end deadlines in ATM networks," in Proceedings of the International Conference on Distributed Computing Systems (ICDCS '95), pp. 60-68, Vancouver, Canada, June 1995.

[264] A. Raha, S. Kamat, X. Jia, and W. Zhao, "Using traffic regulation to meet end-to-end deadlines in ATM networks," IEEE Transactions on Computers, vol. 48, no. 9, pp. 917-935, 1999.

[265] J. K. Ng, S. Song, and W. Zhao, "Integrated end-to-end delay analysis for regulated ATM networks," Real-Time Systems, vol. 25, no. 1, pp. 93-124, 2003.

[266] X. Jia, W. Zhao, and J. Li, "An integrated routing and admission control mechanism for real-time multicast connections in ATM networks," IEEE Transactions on Communications, vol. 49, no. 9, pp. 1515-1519, 2001.

[267] W. Jia and W. Zhao, "Efficient connection admission control algorithms for adaptive QoS real-time connections over ATM networks," European Transactions on Telecommunications, vol. 10, no. 2, pp. 135-151, 1999.

[268] I. Amigo, S. Vaton, T. Chonavel, and F. Larroca, "Maximum delay computation for interdomain path selection," International Journal of Network Management, vol. 22, no. 2, pp. 162179, 2012.

[269] L. Lenzini, E. Mingozzi, and G. Stea, "Delay bounds for FIFO aggregates: a case study," Computer Communications, vol. 28, no. 3, pp. 287-299, 2005.
[270] G. Boggia, P. Camarda, L. A. Grieco, and S. Mascolo, "Feedbackbased bandwidth allocation with call admission control for providing delay guarantees in IEEE 802.11e networks," Computer Communications, vol. 28, no. 3, pp. 325-337, 2005.

[271] M. J. Karam and F. A. Tobagi, "Analysis of delay and delay jitter of voice traffic in the Internet," Computer Networks, vol. 40, no. 6, pp. 711-726, 2002.

[272] H. Fukś, A. T. Lawniczak, and S. Volkov, "Packet Delay in Models of Data Networks," ACM Transactions on Modeling and Computer Simulation, vol. 11, no. 3, pp. 233-250, 2001.

[273] D. E. Wrege, E. W. Knightly, H. Zhang, and J. Liebeherr, "Deterministic delay bounds for VBR video in packet-switching networks: fundamental limits and practical trade-offs," IEEE/ACM Transactions on Networking, vol. 4, no. 3, pp. 352-362, 1996.

[274] J. Liebeherr, D. E. Wrege, and D. Ferrari, "Exact admission control for networks with a bounded delay service," IEEE/ACM Transactions on Networking, vol. 4, no. 6, pp. 885-901, 1996.

[275] S. J. Golestani, "Network delay analysis of a class of fair queueing algorithms," IEEE Journal on Selected Areas in Communications, vol. 13, no. 6, pp. 1057-1070, 1995.

[276] G. Coulouris, J. Dollimore, and T. Kindberg, Distributed Systems: Concepts and Design, Addison-Wesley, New York, NY, USA, 3rd edition, 2001.

[277] W. Zhao, "Challenges in design and implementation of middlewares for real-time systems," Real-Time Systems, vol. 20, no. 2, pp. 115-116, 2001.

[278] S. Davaril, T. Leibfried Jr., S. Natarajan, D. Pruett, L. Sha, and W. Zhao, "Real-time issues in the design of the data management system for space station freedom," in Proceedings of the IEEE Workshop on Real-Time Applications, pp. 161-165, New York, NY, USA, May 1993.

[279] Y. Jiang, Q. Yin, Y. Liu, and S. Jiang, "Fundamental calculus on generalized stochastically bounded bursty traffic for communication networks," Computer Networks, vol. 53, no. 12, pp. 20112021, 2009.

[280] D. Starobinski and M. Sidi, "Stochastically bounded burstiness for communication networks," IEEE Transactions on Information Theory, vol. 46, no. 1, pp. 206-212, 2000.

[281] J. K. Ng, S. Song, and W. Zhao, "Statistical delay analysis on an ATM switch with self-similar input traffic," Information Processing Letters, vol. 74, no. 3, pp. 163-173, 2000.

[282] S. Borst, M. Mandjes, and M. van Uitert, "Generalized processor sharing with light-tailed and heavy-tailed input," IEEE/ACM Transactions on Networking, vol. 11, no. 5, pp. 821-834, 2003.

[283] Y. Liu, C. K. Tham, and Y. Jiang, "A calculus for stochastic QoS analysis," Performance Evaluation, vol. 64, no. 6, pp. 547-572, 2007.

[284] C. Li, A. Burchard, and J. Liebeherr, "A network calculus with effective bandwidth," IEEE/ACM Transactions on Networking, vol. 15, no. 6, pp. 1442-1453, 2007.

[285] Y. Jiang, "A basic stochastic network calculus," ACM SIGCOMM Computer Communication Review, vol. 36, no. 4, pp. 123-134, 2006.

[286] E. Baccarelli, N. Cordeschi, and T. Patriarca, "QoS Stochastic Traffic Engineering for the wireless support of real-time streaming applications," Computer Networks, vol. 56, no. 1, pp. 287-302, 2012.

[287] M. Li and W. Zhao, "Representation of a stochastic traffic bound," IEEE Transactions on Parallel and Distributed Systems, vol. 21, no. 9, pp. 1368-1372, 2010. 
[288] G. A. Korn and T. M. Korn, Mathematical Handbook for Scientists and Engineers, McGraw-Hill, New York, NY, USA.

[289] J. A. Rice, Mathematical Statistics and Data Analysis, Wadsworth Inc., 2nd edition, 1995.

[290] A. A. Borovkov, Probability Theory, Springer, New York, NY, USA, 2013.

[291] T. Field, U. Harder, and P. Harrison, "Network traffic behaviour in switched Ethernet systems," Performance Evaluation, vol. 58, no. 2-3, pp. 243-260, 2004.

[292] A. J. Field, U. Harder, and P. G. Harrison, "Measurement and modelling of self-similar traffic in computer networks," IEE Proceedings: Communications, vol. 151, no. 4, pp. 355-363, 2004.

[293] J. B. Bassingthwaighte, Fractal Physiology, Oxford University Press, New York, NY, USA, 1994. 


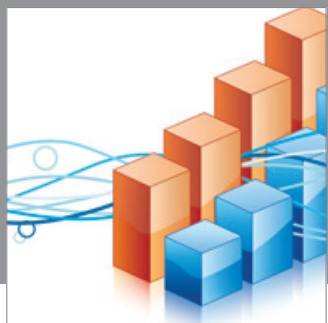

Advances in

Operations Research

mansans

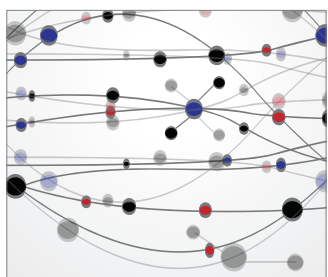

The Scientific World Journal
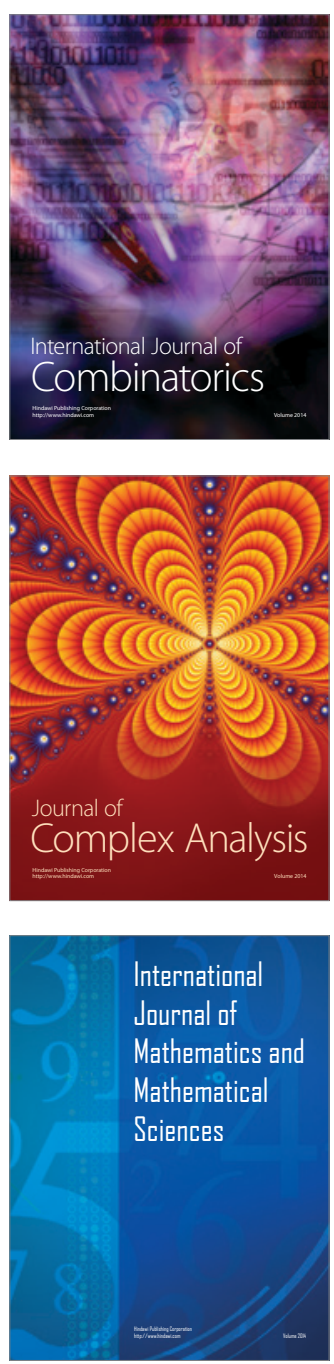
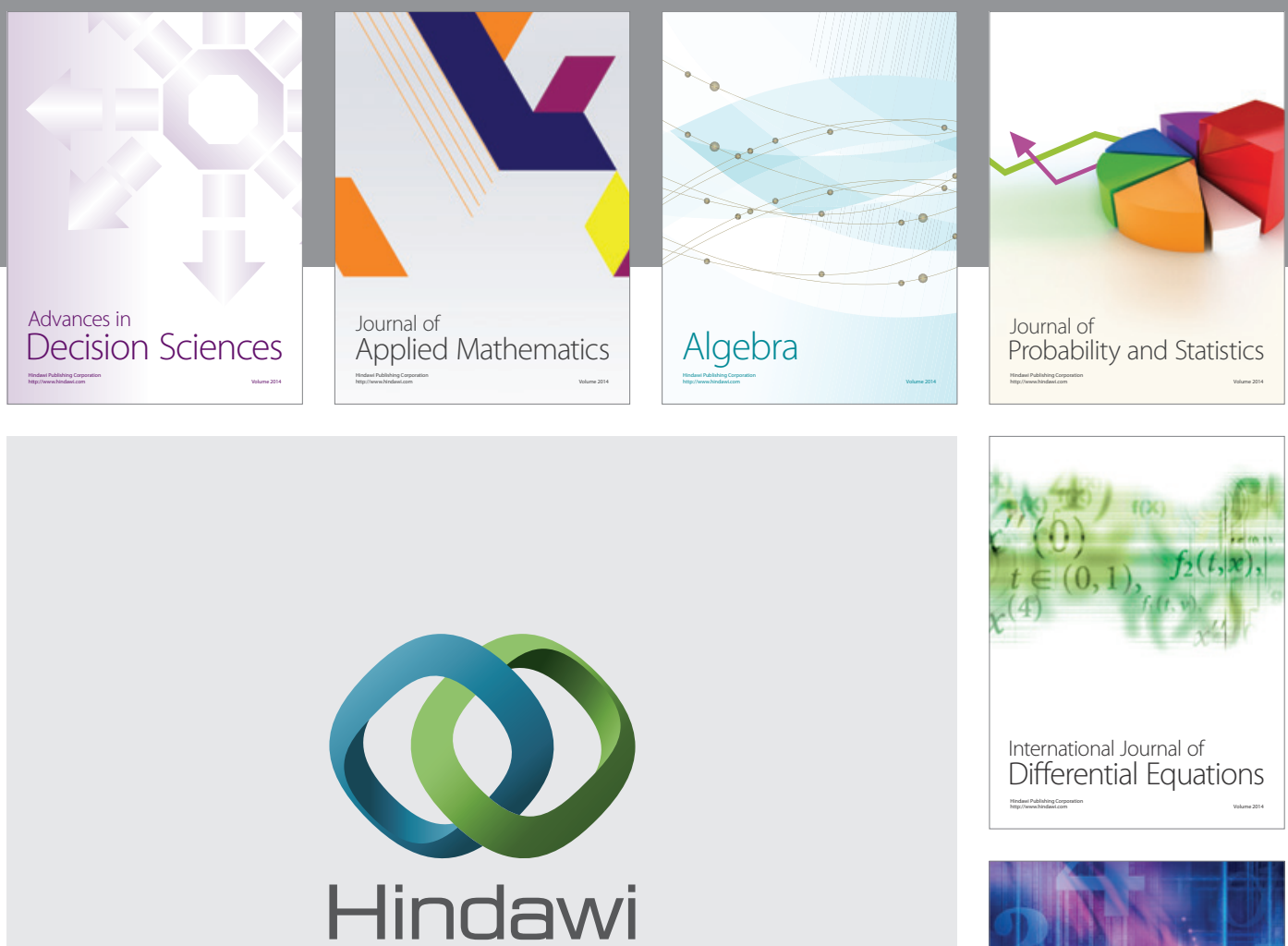

Submit your manuscripts at http://www.hindawi.com
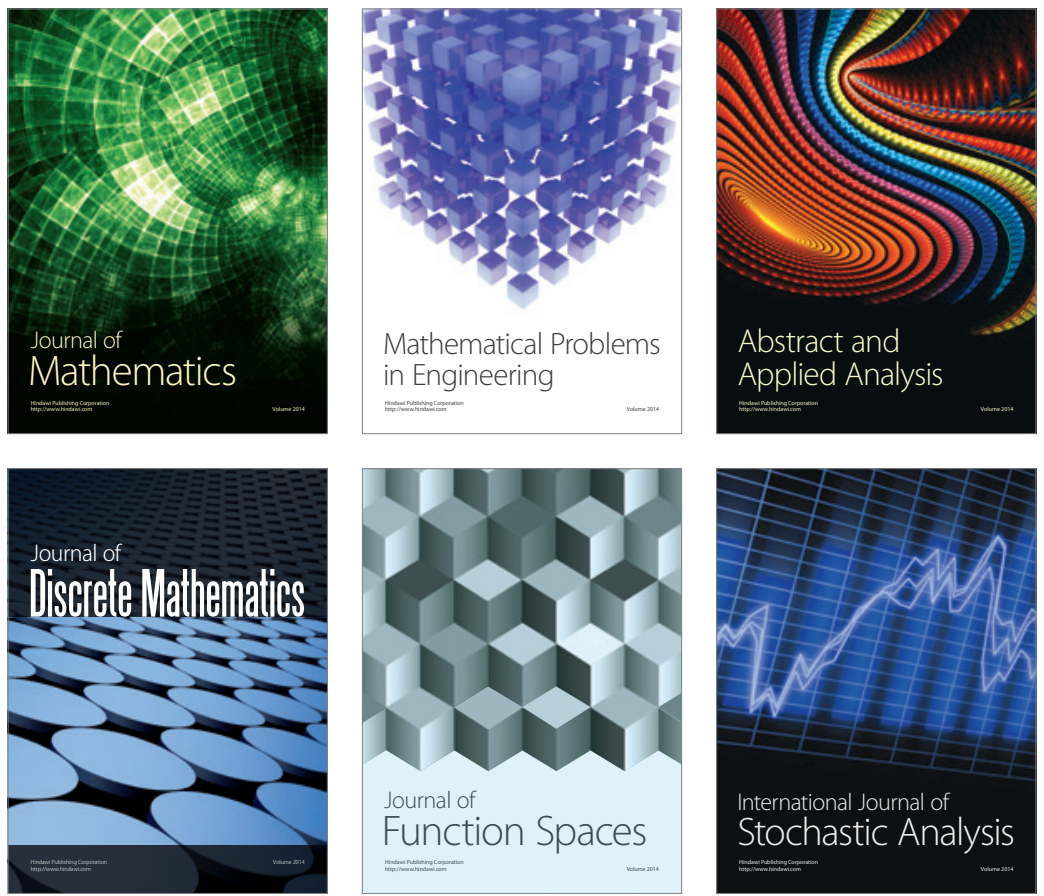

Journal of

Function Spaces

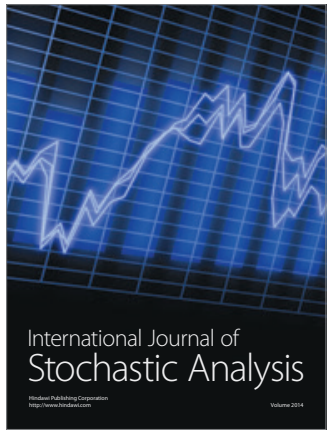

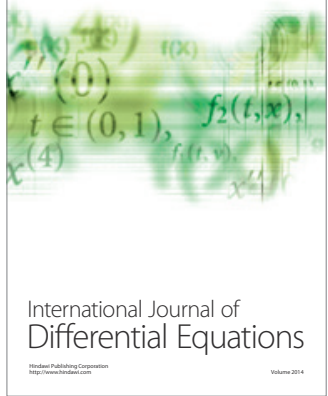
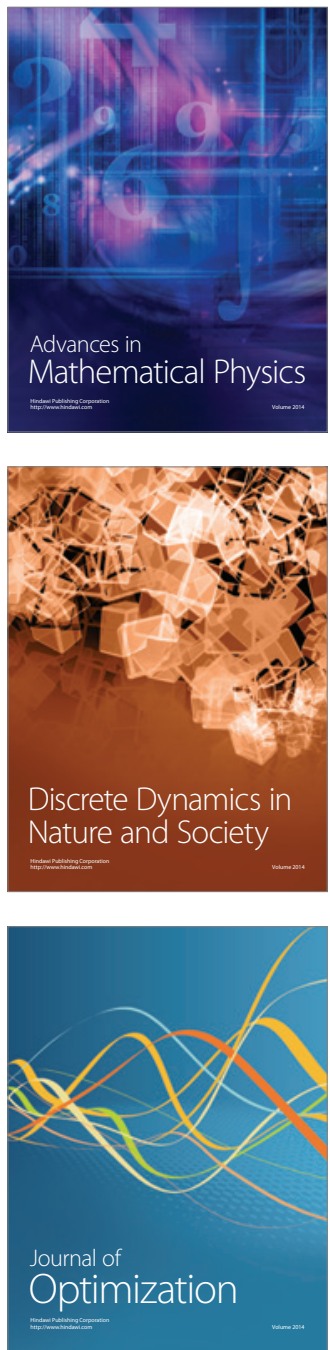